\title{
GIRSANOV AND FEYNMAN-KAC TYPE TRANSFORMATIONS FOR SYMMETRIC MARKOV PROCESSES 해
}

\author{
Zhen-Qing CHEN ${ }^{\text {a }}$, Tu-Sheng ZHANG ${ }^{\text {b }}$ \\ a Department of Mathematics, University of Washington, Seattle, WA 98195, USA \\ ${ }^{\mathrm{b}}$ Department of Mathematics, University of Manchester, Oxford Road, Manchester M13 9PL, UK
}

Received 28 August 2000, revised 10 January 2001

ABSTRACT. - Studied in this paper is the transformation of an arbitrary symmetric Markov process $X$ by multiplicative functionals which are the exponential of continuous additive functionals of $X$ having zero quadratic variations. We characterize the transformed semigroups by their associated quadratic forms. This is done by first identifying the symmetric Markov process under Girsanov transform, which may be of independent interest, and then applying FeynmanKac transform to the Girsanov transformed process. Stochastic analysis for discontinuous martingales is used in our approach. ๑ 2002 Éditions scientifiques et médicales Elsevier SAS

Math. Subj. Class. (1991): Primary 60J45; secondary 60J57; 31C25

RÉSUMÉ. - Dans ce papier, nous étudions la transformation d'un processus symétrique de Markov $X$ par une functionelle multiplicative, qui est l'exponentielle d'une function additive continue, de variation quadratique nulle. Les semi-groupes transformés seront caracterisés par leur formes quadratiques associées. On traite d'abord le cas de la transformation de Girsanov (qui peut avoir un interêt en sai), puis on applique la transformation de Feynman-Kac au processus transformé. L'analyse strochastique pour les martingales discontinues est utilisée dans notre approche. ๑ 2002 Éditions scientifiques et médicales Elsevier SAS

\section{Introduction}

Let $E$ be a Lusin metrizable topological space, i.e., $E$ is homeomorphic to a Borel subset of some compact metric space and $\mathcal{B}(E)$ is the class of Borel sets in $E$. Let $m$ be a $\sigma$-finite measure on $\mathcal{B}(E)$ with $\operatorname{supp}[m]=E$. Let $\chi=\left(\Omega, \mathcal{M}, \mathcal{M}_{t}, X_{t}, \mathbb{P}_{x}, x \in E\right)$ be an $m$-symmetric, right Markov process with state space $E$. In more detail, the rightcontinuous process $[0,+\infty) \ni t \mapsto X_{t}$ is defined on the sample space $(\Omega, \mathcal{M})$, adapted to the filtration $\left(\mathcal{M}_{t}\right)$, and under the law $\mathbb{P}_{x}$ is a strong Markov process with initial

\footnotetext{
The research of the first-named author was supported, in part, by NSF Grant DMS-0071486; that of the second-named author by the Norwegian Research Council.

E-mail addresses: zchen@ math.washington.edu (Z.Q. Chen), tzhang@ maths.man.ac.uk (T.S. Zhang).
} 
condition $X_{0}=x$. The shift operators $\theta_{t}, t \geqslant 0$, satisfy $X_{s} \circ \theta_{t}=X_{s+t}$ identically for $s, t \geqslant 0$. Adjoined to the state space $E$ is an isolated point $\partial \notin E$; the process $X$ retires to $\partial$ at its "lifetime" $\zeta:=\inf \left\{t: X_{t}=\partial\right\}$. Denote $E \cup\{\partial\}$ by $E_{\partial}$.

The transition operators $P_{t}, t \geqslant 0$, are defined by

$$
P_{t} f(x):=\mathbb{E}_{x}\left[f\left(X_{t}\right)\right]=\mathbb{E}_{x}\left[f\left(X_{t}\right) ; t<\zeta\right] .
$$

(Here and in the sequel, unless mentioned otherwise, we use the convention that a function defined on $E$ takes the value 0 at the cemetery point $\partial$.) The $P_{t}$ may be viewed as operators on $L^{2}(E, m)$; as such they form a strongly continuous semigroup of selfadjoint contractions. The associated infinitesimal generator $\mathcal{L}$ is defined by

$$
\mathcal{L} f:=\lim _{t \downarrow 0} t^{-1}\left(P_{t} f-f\right)
$$

on the domain consisting of those $f \in L^{2}(E, m)$ for which the limit in (1.1) exists in the strong sense. The (typically unbounded) operator $-\mathcal{L}$ is self-adjoint and non-negative, so it admits a (self-adjoint, positive) square root $\sqrt{-\mathcal{L}}$. Let $\mathcal{F}$ be the domain of $\sqrt{-\mathcal{L}}$, and define the bilinear form $\mathcal{E}$ on $\mathcal{F}$ by

$$
\mathcal{E}(u, v)=(\sqrt{-\mathcal{L}} u, \sqrt{-\mathcal{L}} v)_{L^{2}(E, m)}, \quad u, v \in \mathcal{F} .
$$

Then $(\mathcal{E}, \mathcal{F})$ is the symmetric Dirichlet form on $L^{2}(E, m)$ associated with the process $X$. It is known (cf. [17]) that $(\mathcal{E}, \mathcal{F})$ is quasi-regular. In fact, there is a one-toone correspondence between symmetric right Markov processes and symmetric quasiregular Dirichlet forms. It is proved in [4] that a Dirichlet form is quasi-regular if and only if it is quasi-homeomorphic to a regular Dirichlet form on a locally compact separable metric space. Thus without loss of generality, we assume throughout this paper that $E$ is a locally compact separable metric space and that $(\mathcal{E}, \mathcal{F})$ is a regular Dirichlet form. Let $\mathcal{F}_{b}=\mathcal{F} \cap L^{\infty}(E, m)$ and denote by $\mathcal{F}_{e}$ the family of $\mathcal{B}(E)$-measurable functions $u$ on $E$ that is finite $m$-a.e. and there is an $\mathcal{E}$-Cauchy sequence $\left\{u_{n}\right\} \subset \mathcal{F}$ such that $\lim _{n \rightarrow \infty} u_{n}=u m$-a.e. on $E$. $\left(\mathcal{E}, \mathcal{F}_{e}\right)$ is called the extended Dirichlet space of $(\mathcal{E}, \mathcal{F})$. Details on symmetric Markov process and Dirichlet form can be found in [12] and [17], including definitions on smooth measures, capacity, $\mathcal{E}$-nest, quasi-continuity, etc.

It is well known (cf. [12]) that for $u \in \mathcal{F}_{e}, u$ has a quasi-continuous version $\widetilde{u}$ and $\widetilde{u}\left(X_{t}\right)$ has the following Fukushima's decomposition:

$$
\widetilde{u}\left(X_{t}\right)=\widetilde{u}\left(X_{0}\right)+M_{t}^{u}+N_{t}^{u}, \quad t \geqslant 0 .
$$

Here $M^{u}$ is a martingale additive functional of $X$ and $N^{u}$ is a continuous additive functional of $X$ having zero quadratic variation. Note that in general $N^{u}$ is not a process of finite variations so $\widetilde{u}\left(X_{t}\right)$ is not a semimartingale, even when $X$ is a Brownian motion. The above decomposition (1.2) can be regarded as an extension of Doob-Meyer decomposition for semimartingales. 
This paper is concerned with the following Feynman-Kac type transformation of $X$ by multiplicative functional $\mathrm{e}^{N_{t}^{u}}$ :

$$
\widehat{P}_{t} f=\mathbb{E}_{x}\left[f\left(X_{t}\right) \mathrm{e}^{N_{t}^{u}}\right] \quad \text { for } f \geqslant 0,
$$

and its characterization.

When $N_{t}^{u}$ is a process of finite variation, (1.3) is a Feynman-Kac transform. Feynman-Kac transforms and Schrödinger operators have been studied extensively by many authors. See for example [5,22] and the references therein. But here $N_{t}^{u}$ is an additive functional of zero energy which does not necessarily have finite variations so the classical results for Feynman-Kac transform do not apply. Here are some interesting examples.

Examples. - Let $X$ be a Brownian motion in $\mathbb{R}$.

(1) (Hilbert transform of Brownian local times) Let $u(x)=x \log |x|-x$, which is locally in the Sobolev space $W^{1,2}(\mathbb{R})$. It is illustrated in Example 5.5.2 of [12] that $t \rightarrow N_{t}^{u}$ is not of finite variation. Furthermore $N_{t}^{u}$ is the value at 0 of the Hilbert transform of Brownian local times, that is,

$$
N_{t}^{u}=\lim _{\varepsilon \downarrow 0} \int_{0}^{t} X_{s}^{-1} 1_{\left\{\left|X_{s}\right| \geqslant \varepsilon\right\}} \mathrm{d} s=\lim _{\varepsilon \downarrow 0} \int_{\mathbb{R}} \frac{L(x, t)}{x} 1_{\{|x| \geqslant \varepsilon\}} \mathrm{d} x .
$$

Here $L(x, t)$ is the local time of $X$ at $x$. If we define for $a \in \mathbb{R}, u_{a}(x)=u(x-a)$, then

$$
N_{t}^{u_{a}}=\lim _{\varepsilon \downarrow 0} \int_{0}^{t}\left(X_{s}-a\right)^{-1} 1_{\left\{\left|X_{s}-a\right| \geqslant \varepsilon\right\}} \mathrm{d} s=\lim _{\varepsilon \downarrow 0} \int_{\mathbb{R}} \frac{L(x, t)}{x-a} 1_{\{|x-a| \geqslant \varepsilon\}} \mathrm{d} x .
$$

(2) (Fractional derivative of Brownian local times) For $u=\frac{|x|^{1-\alpha}}{\alpha(\alpha+1)} \operatorname{sgn}(x)$ with $\alpha \in$ $(0,1 / 2), t \rightarrow N_{t}^{u}$ is not of finite variations and

$$
N_{t}^{u}=\lim _{\varepsilon \downarrow 0} \int_{0}^{t} X_{s}\left|X_{s}\right|^{-\alpha-2} 1_{\left\{\left|X_{s}\right| \geqslant \varepsilon\right\}} \mathrm{d} s=\int_{0}^{\infty} \frac{L(x, t)-L(-x, t)}{x^{1+\alpha}} \mathrm{d} x,
$$

which is the value at 0 of the symmetric fractional derivative of order $\alpha$ for the Brownian local time (see [26] and [27]).

One can similarly make examples for one-dimensional symmetric stable processes as well (cf. [10]).

The above examples demonstrates the additive functional $N^{u}$ of zero energy in (1.2) contains many important as well as interesting continuous additive functionals and therefore it is worthwhile to investigate the Feynman-Kac type transform (1.3) by $\mathrm{e}^{N_{t}^{u}}$. To state the main result of this paper, we need the following definition. 
DEFINITION 1.1. - A smooth measure $\mu$ is said to be in Kato class of process $X$ if its associated PCAF $A_{t}$ satisfies condition

$$
\lim _{t \downarrow 0} \operatorname{esssup}_{x \in E} \mathbb{E}_{x}\left[A_{t}\right]=0
$$

Here esssup is the abbreviation for

$x \in E$

$$
\inf _{\substack{N \subset E \\ \operatorname{Cap}_{1}(N)=0}} \sup _{x \in E \backslash N}
$$

where $\mathrm{Cap}_{1}$ denotes the 1-capacity of $X$. Similarly, we define $\underset{x \in E}{\operatorname{essinf}}$ to be

$$
\inf _{\substack{N \subset E \\ \operatorname{Cap}_{1}(N)=0}} \inf _{x \in E \backslash N} .
$$

Let $\left\langle M^{u}\right\rangle$ be the predictable dual projection of the square bracket $\left[M^{u}\right]$ of $M^{u}$ in (1.2), which is a positive continuous additive functional (PCAF in abbreviation) of $X$. We denote by $\mu_{\langle u\rangle}$ the Revuz measure of $\left\langle M^{u}\right\rangle$. Measure $\mu_{\langle u\rangle}$ is called the energy measure of $u$.

THEOREM 1.2. - Assume that function $u$ is in $\mathcal{F}_{e}$ such that $\mu_{\langle u\rangle}$ is in Kato class of $X$. Then $\widehat{P}_{t}$ is a strongly continuous symmetric semigroup on $L^{2}(E, m)$. Let $(Q, \mathcal{D}(Q))$ be the quadratic form associated with $\widehat{P}_{t}$ on $L^{2}(E, m)$. Then $\mathcal{D}(Q)=\mathcal{F}$ and for $f, g \in \mathcal{F}_{b}$,

$$
Q(f, g)=\mathcal{E}(f, g)+\mathcal{E}(u, f g) .
$$

Energy measure $\mu_{\langle u\rangle}$ can be calculated through formulas (2.1)-(2.2) in next section. Sufficient conditions for being in the Kato class of Brownian motion, symmetric stable processes, a large family of Lévy processes, and processes with relativistic Hamiltonian generators can be found in [5] and [28]. Here we just mention one example. Let $X$ be a symmetric diffusion in $\mathbb{R}^{n}$ with infinitesimal generator $\mathcal{L}=\frac{1}{2} \sum_{i, j=1}^{n} \frac{\partial}{\partial x_{i}}\left(a_{i j}(x) \frac{\partial}{\partial x_{j}}\right)$, where matrix $\left(a_{i j}(x)\right)_{1 \leqslant i, j \leqslant n}$ is uniformly elliptic and bounded, that is, there is $\lambda>1$ such that for $m$-a.e. $x \in \mathbb{R}^{n}$ and $\xi=\left(\xi_{1}, \ldots, \xi_{n}\right) \in \mathbb{R}^{n}$,

$$
\lambda^{-1}\|\xi\|^{2} \leqslant \sum_{i, j=1}^{n} a_{i j}(x) \xi_{i} \xi_{j} \leqslant \lambda\|\xi\|^{2}
$$

The Dirichlet form $(\mathcal{E}, \mathcal{F})$ in $L^{2}\left(\mathbb{R}^{n}, \mathrm{~d} x\right)$ for $X$ is: $\mathcal{F}=W^{1,2}\left(\mathbb{R}^{n}\right)=\left\{f \in L^{2}\left(\mathbb{R}^{n}, \mathrm{~d} x\right)\right.$ : $\left.\nabla f \in L^{2}\left(\mathbb{R}^{n}, \mathrm{~d} x\right)\right\}$ and

$$
\mathcal{E}(f, g)=\frac{1}{2} \int_{\mathbb{R}^{n}} \sum_{i, j=1}^{n} a_{i j}(x) \frac{\partial f}{\partial x_{i}} \frac{\partial g}{\partial x_{j}} \mathrm{~d} x, \quad f, g \in W^{1,2}\left(\mathbb{R}^{n}\right) .
$$

The extended Dirichlet space $\mathcal{F}_{e}=\left\{f \in L_{\mathrm{loc}}^{2}\left(\mathbb{R}^{n}, \mathrm{~d} x\right): \nabla f \in L^{2}\left(\mathbb{R}^{n}, \mathrm{~d} x\right)\right\}$ (cf. Example 1.5.2 of [12]). Note that for $u \in \mathcal{F}_{e}$, its energy measure is $\mu_{\langle u\rangle}(\mathrm{d} x)=$ 
$\sum_{i, j=1}^{n} a_{i j}(x) \frac{\partial u}{\partial x_{i}} \frac{\partial u}{\partial x_{j}} \mathrm{~d} x$. Thus a locally $L^{2}$-integrable function $u$ with $\nabla u \in L^{2}\left(\mathbb{R}^{n}, \mathrm{~d} x\right) \cap$ $L^{p}\left(\mathbb{R}^{n}, \mathrm{~d} x\right)$ for some $p>n$ is a function in $\mathcal{F}_{e}$ with $\mu_{\langle u\rangle}$ in the Kato class of $X$ and therefore Theorem 1.2 applies.

When $X$ is Brownian motion on $\mathbb{R}^{n}$, Theorem 1.2 was established by Glover et al in [14] under an additional assumption that $u$ is a bounded function in $\mathcal{F}=W^{1,2}\left(\mathbb{R}^{n}\right)$ using an approximation method that employed some special properties of Brownian motion. Zhang [25] studied the problem for symmetric Lévy processes and bounded $u$, also by an approximation method, where the property of stationary independent increments for Lévy processes is used in an essential way.

The approach in this paper is more direct and our results are applicable to arbitrary symmetric right Markov processes. Let us explain our idea behind our method. We first establish our result for bounded function $u \in \mathcal{F}_{e}$ whose energy measure $\mu_{\langle u\rangle}$ is in Kato class of $X$. In view of (1.2), we have

$$
\widehat{P}_{t} f(x)=\mathbb{E}_{x}\left[f\left(X_{t}\right) \mathrm{e}^{\widetilde{u}\left(X_{t}\right)-\widetilde{u}\left(X_{0}\right)-M_{t}^{u}}\right]=\mathrm{e}^{-\widetilde{u}(x)} \mathbb{E}_{x}\left[\left(f \mathrm{e}^{\widetilde{u}}\right)\left(X_{t}\right) \mathrm{e}^{-M_{t}^{u}}\right] .
$$

When $X$ has continuous sample paths,

$$
L_{t}=\exp \left(-M_{t}^{u}-\frac{1}{2}\left\langle M^{u}\right\rangle_{t}\right)
$$

is an exponential martingale. Since

$$
\widehat{P}_{t} f(x)=\mathrm{e}^{-\widetilde{u}(x)} \mathbb{E}_{x}\left[L_{t} \exp \left(\frac{1}{2}\left\langle M^{u}\right\rangle_{t}\right)\left(f \mathrm{e}^{\widetilde{u}}\right)\left(X_{t}\right)\right],
$$

the transform (1.3) is the result of a Girsanov transform by exponential martingale $L_{t}$ followed by a Feynman-Kac transform $\exp \left(\frac{1}{2}\left\langle M^{u}\right\rangle_{t}\right)$. In the general case, $X$ may have jumps and killings so things become much more involved but the same idea still works. Our key observation is that when $X$ is a general symmetric Markov process,

$$
\exp \left(-M_{t}^{u}\right)=L_{t} \exp \left(A_{t}\right)
$$

where $L_{t}$ is an exponential martingale defined by

$$
L_{t}=1+\int_{0}^{t} L_{s-} \mathrm{e}^{\widetilde{u}\left(X_{s-}\right)} \mathrm{d} M_{s}^{\mathrm{e}^{-u}}
$$

with $M_{s}^{\mathrm{e}^{-u}}:=M_{s}^{\mathrm{e}^{-u}-1}$, and $A_{t}$ is a continuous additive functional of $X$ having finite variations. So the key to study the transformation (1.3) is to study Girsanov transform by exponential martingale $L_{t}$ and identify the transformed process. Once this is done, it can be shown that under the condition of Theorem 1.2, the Revuz measure $\mu$ of $A_{t}$ has property that $\mu^{+}$is in Kato class of the Girsanov transformed process and hence results from [1] can be applied. 
We show that under the condition that $u$ is a bounded function in $\mathcal{F}_{e}$, exponential local martingale $L_{t}$ defines a family of probability measures $\left\{\widetilde{P}_{x}, x \in E\right\}$ through

$$
\mathrm{d} \widetilde{P}_{x}=L_{t} \mathrm{~d} P_{x} \quad \text { on } \mathcal{M}_{t}, x \in E .
$$

We will characterize the transformed process $\widetilde{X}_{t}$ under $\widetilde{\mathbb{P}}_{x}, x \in E$, by identifying its associated Dirichlet form. Our method of identifying the Dirichlet form is influenced by Fitzsimmons [9]. However, difficulties and delicacy arise due to the possible jumps and the killings of the process $X$. Once Theorem 1.2 is established for bounded $u \in \mathcal{F}_{e}$, we extend it to general $u \in \mathcal{F}_{e}$ by approximating it with $u_{n}=((-n) \vee u) \wedge n$. Here for two real numbers $a$ and $b, a \vee b:=\max \{a, b\}$ and $a \wedge b:=\min \{a, b\}$.

Girsanov transform of Brownian motion and other Markov processes by supermartingale multiplicative functionals has been studied by physicists as well as mathematicians, including names of Cameron and Martin, Maruyama, Girsanov, Kac, Darling and Siegert, Hunt, Dynkin, ..., for many years. See the Notes and Comments of Blumenthal and Getoor [3] for a brief history and the references therein. The one that is closest to our Girsanov transform result in this paper is the work of Fukushima and Takeda [13] and Fitzsimmons [9]. In [13], transformation by (1.6) of a symmetric Markov process is considered, but with $\mathrm{e}^{-u}$ being replaced by a positive function $\rho$ in the domain of generator $\mathcal{L}$. In [9], transformation of symmetric diffusions $X$ without killings by exponential local martingale (1.5) for positive $u$ such that $\mathrm{e}^{-u} \in \mathcal{F}_{\text {loc }}$ is considered and the Dirichlet form for the transformed process is identified. For other related work on transformation by supermartingale multiplicative functional in the context of symmetric diffusions and local Dirichlet forms, see the references in [9], [12] and [23].

The Girsanov transform studied in this paper is for an arbitrary symmetric Markov process $X$ transformed by a supermartingale related to function $\mathrm{e}^{-u}$ that is not in the domain of the generator $\mathcal{L}$ of $X$. A new feature of our result under transformation (1.6) is that the killings of the original process $X$ do not disappear after the transformation as oppose to the case in [13]. In fact the new transformed process has killing measure $\mathrm{e}^{-u(x)} \kappa(\mathrm{d} x)$ (rather than $\mathrm{e}^{-2 u(x)} \kappa(\mathrm{d} x)$ as one might think), where $\kappa(\mathrm{d} x)$ is the killing measure of $X$. Another interesting feature is that the time-reversal technique and LyonsZheng's forward-backward martingale decomposition technique work equally effective for symmetric Markov processes with possible jumps and killings. Our method can be modified to recover and extend the Girsanov transform result in Fukushima and Takeda [13]. Details on this will appear in a separate paper.

A closely related but somewhat inverse question is, given an $m$-symmetric Markov process $X$, can one characterize all $v$-symmetric Markov process $Y$ whose law is absolutely continuous to that of $X$. The research on the latter problem was initiated by Orey [18] in 1974, where $X$ is one-dimensional Brownian motion. Fukushima [11] studied the case for multidimensional Brownian motion, and Oshima [19] for a special class of diffusions. Fitzsimmons [9] treated general symmetric diffusions without killings. We plan to study the absolutely continuity problem for general symmetric right Markov processes in a separate paper.

The rest of the paper is organized as follows. After Section 2 on preliminaries, the aforementioned results for Girsanov transform by $L_{t}$ in (1.6) were established in Section 3. Theorem 1.2 is proved in Section 4. 


\section{Preliminaries}

Recall that we assumed that $E$ is a locally compact separable metric space and the Dirichlet form $(\mathcal{E}, \mathcal{F})$ in $L^{2}(E, m)$ is regular, i.e., $\mathcal{F} \cap C_{c}(E)$ is dense both in $\mathcal{F}$ with respect to the $\mathcal{E}_{1}(\cdot, \cdot)=\mathcal{E}(\cdot, \cdot)+(\cdot, \cdot)$ norm and in $C_{c}(E)$, the space of continuous functions with compact supports, with respect to the uniform norm. Therefore $\chi=$ $\left(\Omega, \mathcal{M}, \mathcal{M}_{t}, X_{t}, \mathbb{P}_{x}, x \in E\right)$ can be taken as a Hunt process on $E$. For $\alpha>0$, let $G_{\alpha}=\int_{0}^{\infty} \mathrm{e}^{-\alpha t} P_{t} \mathrm{~d} t$ be the $\alpha$-resolvent of $X$. When $\mu$ is a smooth measure, we use $U_{1} \mu$ to denote the 1-potential of $\mu$. If $\mu(\mathrm{d} x)=f(x) m(\mathrm{~d} x)$, then $U_{1} \mu=G_{1} f$.

Let $\left(N(x, \mathrm{~d} y), H_{s}\right)$ be the Lévy system for $X$. If we use $v$ to denote the Revuz measure of the PCAF $H$, then (cf. [12]) the jumping measure $J$ and the killing measure $\kappa$ of $X$ are given by

$$
J(\mathrm{~d} x, \mathrm{~d} y)=\frac{1}{2} N(x, \mathrm{~d} y) \nu(\mathrm{d} x) \quad \text { and } \quad \kappa(\mathrm{d} x)=N(x, \partial) \nu(\mathrm{d} x) .
$$

Furthermore the following Beurling-Deny decomposition holds for $f, g \in \mathcal{F}_{e}$,

$$
\begin{aligned}
\mathcal{E}(f, g)= & \mathcal{E}^{(c)}(f, g)+\int_{E \times E \backslash d}(\widetilde{f}(x)-\widetilde{f}(y))(\widetilde{g}(x)-\widetilde{g}(y)) J(\mathrm{~d} x, \mathrm{~d} y) \\
& +\int_{E} \widetilde{f}(x) \widetilde{g}(x) \kappa(\mathrm{d} x),
\end{aligned}
$$

where bilinear form $\mathcal{E}^{c}$ is the strongly local part of $\mathcal{E}$.

The martingale part $M_{t}^{u}$ in (1.2) can be decomposed as

$$
M_{t}^{u}=M_{t}^{u, c}+M_{t}^{u, j}+M_{t}^{u, k},
$$

where

$$
\begin{aligned}
M_{t}^{u, j}= & \lim _{n \rightarrow \infty}\left\{\sum_{0<s \leqslant t}\left(\widetilde{u}\left(X_{s}\right)-\widetilde{u}\left(X_{s-}\right)\right) 1_{\left\{\left|\widetilde{u}\left(X_{s}\right)-\widetilde{u}\left(X_{s-}\right)\right|>1 / n\right\}} 1_{\{t<\zeta\}}\right. \\
& \left.-\int_{0}^{t}\left(\int_{\left\{y \in E:\left|\widetilde{u}(y)-\widetilde{u}\left(X_{s}\right)\right|>1 / n\right\}}\left(\widetilde{u}(y)-\widetilde{u}\left(X_{s}\right)\right) N\left(X_{s}, \mathrm{~d} y\right)\right) \mathrm{d} H_{s}\right\}, \\
M_{t}^{u, k}= & \int_{0}^{t} \widetilde{u}\left(X_{s}\right) N\left(X_{s}, \partial\right) \mathrm{d} H_{s}-\widetilde{u}\left(X_{\zeta-}\right) 1_{\{t \geqslant \zeta\},}
\end{aligned}
$$

and $M_{t}^{u, c}$ are respectively the jumping, killing and the continuous part of martingale $M^{u}$. The limit in the expression for $M^{u, k}$ is in the sense of in probability and in the norm of space of square integrable martingales (cf. [12]).

Let $\mu_{\langle u\rangle}, \mu_{\langle u\rangle}^{c}, \mu_{\langle u\rangle}^{j}$ and $\mu_{\langle u\rangle}^{k}$ be the smooth Revuz measures associated with the sharp bracket PCAF $\left\langle M^{u}\right\rangle,\left\langle M^{u, c}\right\rangle,\left\langle M^{u, j}\right\rangle$ and $\left\langle M^{u, k}\right\rangle$, respectively. Then,

$$
\mu_{\langle u\rangle}(\mathrm{d} x)=\mu_{\langle u\rangle}^{c}(\mathrm{~d} x)+\mu_{\langle u\rangle}^{j}(\mathrm{~d} x)+\mu_{\langle u\rangle}^{k}(\mathrm{~d} x),
$$


where $\mu_{\langle u\rangle}^{c}$ satisfies $\mu_{\langle u\rangle}^{c}(E)=2 \mathcal{E}^{c}(u, u)$,

$$
\mu_{\langle u\rangle}^{j}(\mathrm{~d} x)=2 \int_{E}(\widetilde{u}(x)-\widetilde{u}(y))^{2} J(\mathrm{~d} x, \mathrm{~d} y), \quad \text { and } \quad \mu_{\langle u\rangle}^{k}(\mathrm{~d} x)=\widetilde{u}(x)^{2} \kappa(\mathrm{d} x) .
$$

Let $u_{n}=((-n) \vee u) \wedge n$. By Theorem 5.2.3 of [12], $\mu_{\left\langle u_{n}\right\rangle}$ satisfies

$$
\int_{E} \tilde{f}(x) \mu_{\left\langle u_{n}\right\rangle}(\mathrm{d} x)=2 \mathcal{E}\left(u_{n} f, u_{n}\right)-\mathcal{E}\left(u_{n}^{2}, f\right) \quad \text { for any bounded } f \in \mathcal{F}_{e},
$$

which can be used to find the expression of $\mu_{\left\langle u_{n}\right\rangle}$ and therefore of $\mu_{\langle u\rangle}=\lim _{n \rightarrow \infty} \mu_{\left\langle u_{n}\right\rangle}$. Note that

$$
\sup _{t>0} \frac{1}{t} \mathbb{E}_{m}\left[\left(M_{t}^{u}\right)^{2}\right]=\lim _{t \downarrow 0} \frac{1}{t} \mathbb{E}_{m}\left\langle M^{u}\right\rangle_{t}=\mu_{\langle u\rangle}(E)=\mathcal{E}(u, u)-\frac{1}{2} \int_{E} \widetilde{u}(x)^{2} \kappa(\mathrm{d} x) .
$$

In particular,

$$
\mathbb{E}_{m}\left[\left(M_{t}^{u}\right)^{2}\right] \leqslant t \mathcal{E}(u, u) \quad \text { for all } t>0 \text { and } u \in \mathcal{F}_{e} .
$$

We now present some results which will be used in the sequel. First recall Proposition 3.1 of $[1]$ :

LEMMA 2.1. - If $\mu$ is in the Kato class, then for any $\varepsilon>0$, there is a constant $A_{\varepsilon}>0$ such that

$$
\int_{E} \widetilde{h}(x)^{2} \mu(\mathrm{d} x) \leqslant \varepsilon \mathcal{E}(h, h)+A_{\varepsilon} \int_{E} h(x)^{2} m(\mathrm{~d} x), \quad h \in \mathcal{F} .
$$

Define a bilinear form $Q$ on $\mathcal{F}_{b}$ by

$$
Q(f, g)=\mathcal{E}(f, g)+\mathcal{E}(u, f g), \quad f, g \in \mathcal{F}_{b} .
$$

Proposition 2.2. - Assume that $u \in \mathcal{F}_{e}$ whose energy measure $\mu_{\langle u\rangle}$ is in Kato class of $X$.

(i) The quadratic form $\left(Q, \mathcal{F}_{b}\right)$ is well defined and lower bounded. More precisely, there are constants $\alpha>0$ and $\lambda>1$ such that for every $f \in \mathcal{F}_{b}$,

$$
\lambda^{-1} \mathcal{E}_{1}(f, f) \leqslant Q(f, f)+\alpha \int_{E} f^{2}(x) m(\mathrm{~d} x) \leqslant \lambda \mathcal{E}_{1}(f, f) .
$$

(ii) Let $(Q, \mathcal{D}(Q))$ be the smallest closed extension of $\left(Q, \mathcal{F}_{b}\right)$. Then $\mathcal{D}(Q)=\mathcal{F}$ and for $f, g \in \mathcal{F}$,

$$
\begin{aligned}
& Q(f, g)=\mathcal{E}(f, g)+\frac{1}{2} \int \widetilde{f}(x) \mu_{\langle u, g\rangle}^{c}(\mathrm{~d} x)+\frac{1}{2} \int \widetilde{g}(x) \mu_{\langle u, f\rangle}^{c}(\mathrm{~d} x) \\
& \quad+\int_{E \times E \backslash d}(\widetilde{f}(x) \widetilde{g}(x)-\widetilde{f}(y) \widetilde{g}(y))(\widetilde{u}(x)-\widetilde{u}(y)) J(\mathrm{~d} x, \mathrm{~d} y)+\int \widetilde{u}(x) \widetilde{f}(x) \widetilde{g}(x) \kappa(\mathrm{d} x) .
\end{aligned}
$$


Proof. - (i) Since $\mu_{\langle u\rangle}$ is in Kato class, for any $\varepsilon>0$, there is a constant $A_{\varepsilon}$ such that

$$
\int_{E} \widetilde{h}(x)^{2} \mu_{\langle u\rangle}(\mathrm{d} x) \leqslant \varepsilon \mathcal{E}(h, h)+A_{\varepsilon} \int_{E} h(x)^{2} m(\mathrm{~d} x), \quad h \in \mathcal{F} .
$$

Note that for $f, g \in \mathcal{F}$,

$$
\begin{aligned}
\left|\int_{E} \widetilde{f}(x) \mu_{\langle u, g\rangle}^{c}(\mathrm{~d} x)\right| & \leqslant\left(\int_{E} \widetilde{f}(x)^{2} \mu_{\langle u\rangle}^{c}(\mathrm{~d} x)\right)^{1 / 2}\left(\int_{E} \mu_{\langle g\rangle}^{c}(\mathrm{~d} x)\right)^{1 / 2} \\
& \leqslant \int_{E} \widetilde{f}(x)^{2} \mu_{\langle u\rangle}^{c}(\mathrm{~d} x)+\frac{1}{4} \mathcal{E}^{(c)}(g, g) .
\end{aligned}
$$

For the jumping part, by the symmetry of $J$ we have

$$
\begin{aligned}
&\left|\int_{E \times E \backslash d}(\widetilde{u}(x)-\widetilde{u}(y))(\tilde{f}(x)-\widetilde{f}(y))(\widetilde{g}(x)+\widetilde{g}(y)) J(\mathrm{~d} x, \mathrm{~d} y)\right| \\
& \leqslant\left(\int_{E \times E \backslash d}(\widetilde{f}(x)-\widetilde{f}(y))^{2} J(\mathrm{~d} x, \mathrm{~d} y)\right)^{1 / 2} \\
& \times\left(\int_{E \times E \backslash d}(\widetilde{u}(x)-\widetilde{u}(y))^{2}(\widetilde{g}(x)+\widetilde{g}(y))^{2} J(\mathrm{~d} x, \mathrm{~d} y)\right)^{1 / 2} \\
& \leqslant 2\left(\int_{E \times E \backslash d}(\widetilde{f}(x)-\widetilde{f}(y))^{2} J(\mathrm{~d} x, \mathrm{~d} y)\right)^{1 / 2} \\
& \times\left(\int_{E \times E \backslash d} \widetilde{g}(x)^{2}(\widetilde{u}(x)-\widetilde{u}(y))^{2} J(\mathrm{~d} x, \mathrm{~d} y)\right)^{1 / 2} \\
& \leqslant \frac{1}{2} \int_{E \times E \backslash d}(\widetilde{f}(x)-\widetilde{f}(y))^{2} J(\mathrm{~d} x, \mathrm{~d} y)+2 \int_{E} \widetilde{g}(x)^{2} \mu_{\langle u\rangle}^{j}(\mathrm{~d} x) .
\end{aligned}
$$

Observe also that

$$
\begin{aligned}
\left|\int_{E} \widetilde{u}(x) \widetilde{f}(x) \widetilde{g}(x) \kappa(\mathrm{d} x)\right| & \leqslant\left(\int_{E} \widetilde{f}(x)^{2} \widetilde{u}(x)^{2} \kappa(\mathrm{d} x)\right)^{1 / 2}\left(\int_{E} \widetilde{g}(x)^{2} \kappa(\mathrm{d} x)\right)^{1 / 2} \\
& \leqslant \frac{1}{2} \int_{E} \widetilde{f}(x)^{2} \mu_{\langle u\rangle}^{k}(\mathrm{~d} x)+\frac{1}{2} \int_{E} \widetilde{g}(x)^{2} \kappa(\mathrm{d} x) .
\end{aligned}
$$

As $\mu_{\langle u\rangle}=\mu_{\langle u\rangle}^{c}+\mu_{\langle u\rangle}^{j}+\mu_{\langle u\rangle}^{k}$, applying (2.6) to (2.7)-(2.9) with $f=g \in \mathcal{F}_{b}$, we see that there exists a constant $A$ such that

$$
\left|\mathcal{E}\left(u, f^{2}\right)\right| \leqslant \frac{2}{3} \mathcal{E}(f, f)+A \int_{E} f(x)^{2} m(\mathrm{~d} x) .
$$


This proves (2.4).

(ii) Clearly (2.5) holds for $f, g \in \mathcal{F}_{b}$ and (2.4) implies that $\mathcal{D}(Q)=\mathcal{F}$. For general $f, g \in \mathcal{F}$, let $f_{n}=((-n) \vee f) \wedge n$ and $g_{n}=((-n) \vee g) \wedge n$. As $f_{n} \rightarrow f$ and $g_{n} \rightarrow g$ with respect to the $\mathcal{E}_{1}$-norm, $Q(f, g)=\lim _{n \rightarrow \infty} Q\left(f_{n}, g_{n}\right)$ and (2.5) follows immediately from (2.6)-(2.9).

DEFINITION 2.3. $-A$ (non-negative) smooth measure $\mu$ is said to be of finite $\mathcal{E}$ energy integral if there is a constant $c>0$ such that

$$
\int_{E} \tilde{f}(x) \mu(\mathrm{d} x) \leqslant c \sqrt{\mathcal{E}_{1}(f, f)} \quad \text { for all } f \in \mathcal{F} .
$$

LEMma 2.4. - Assume that $\mu$ is of finite $\mathcal{E}$-energy integral and $A_{t}$ is its associated $P C A F$. Then $h^{t}(x):=\mathbb{E}_{x}\left[A_{t}\right]$ is quasi-continuous.

Proof. - For any $\alpha>0$, define $h_{\alpha}(x)=\mathbb{E}_{x}\left[\int_{0}^{t} \mathrm{e}^{-\alpha s} \mathrm{~d} A_{s}\right]$. Then

$$
h_{\alpha}(x)=\mathbb{E}_{x}\left[\int_{0}^{\infty} \mathrm{e}^{-\alpha s} \mathrm{~d} A_{s}\right]-\mathrm{e}^{-\alpha t} \mathbb{E}_{x}\left[\mathbb{E}_{X_{t}}\left[\int_{0}^{\infty} \mathrm{e}^{-\alpha s} \mathrm{~d} A_{s}\right]\right]
$$

is quasi-continuous and $\lim _{\alpha \rightarrow 0} h_{\alpha}(x)=h^{t}(x)$ for q.e. $x \in E$. By Theorem 2.1.4 in [12], it is sufficient to show that $\sup _{\alpha \leqslant 1} \mathcal{E}\left(h_{\alpha}, h_{\alpha}\right)<\infty$. By the proof of Lemma 5.1.9 in [12], we see that $h^{t}(x) \in \mathcal{F}$ and

$$
\mathcal{E}\left(h^{t}, h^{t}\right) \leqslant\left\langle\mu, h^{t}\right\rangle \leqslant \mathrm{e}^{t}\left\langle\mu, U_{1} \mu\right\rangle \leqslant \mathrm{e}^{t} \mathcal{E}_{1}\left(U_{1} \mu, U_{1} \mu\right), \quad t>0 .
$$

By integration by parts,

$$
h_{\alpha}(x)=\mathrm{e}^{-\alpha t} h^{t}(x)-\alpha \int_{0}^{t} h^{s}(x) \mathrm{e}^{-\alpha s} \mathrm{~d} s .
$$

Thus for $0<\alpha \leqslant 1$,

$$
\begin{aligned}
\mathcal{E}\left(h_{\alpha}, h_{\alpha}\right) & \leqslant 2 \mathrm{e}^{-2 \alpha t} \mathcal{E}\left(h^{t}, h^{t}\right)+2 \alpha^{2} \int_{0}^{t} \mathrm{e}^{-2 \alpha s} \mathcal{E}\left(h^{s}, h^{s}\right) \mathrm{d} s \\
& \leqslant\left(2 \mathrm{e}^{-2 \alpha t} \mathrm{e}^{t}+2 \alpha^{2} \int_{0}^{t} \mathrm{e}^{2(1-\alpha) s} \mathrm{~d} s\right) \mathcal{E}_{1}\left(U_{1} \mu, U_{1} \mu\right) \\
& \leqslant\left(2 \mathrm{e}^{t}+\mathrm{e}^{2 t}\right) \mathcal{E}_{1}\left(U_{1} \mu, U_{1} \mu\right),
\end{aligned}
$$

which implies that $\sup _{\alpha \leqslant 1} \mathcal{E}\left(h_{\alpha}, h_{\alpha}\right)<\infty$. This completes the proof.

COROLlaRY 2.5. - Assume that $\mu$ is a smooth measure with $\mu(E)<\infty$. Then $\mu$ is in Kato class if and only if there is a properly exceptional set $N$ such that

$$
\lim _{t \rightarrow 0} \sup _{x \in E \backslash N} \mathbb{E}_{x}\left[A_{t}\right]=0 .
$$


Proof. - Clearly if (2.10) holds, then $\mu$ is in Kato class. Now assume that $\mu$ is in Kato class. Since $\mu(E)<\infty$, by Cauchy-Schwartz and Lemma 2.1, we see that $\mu$ is of finite $\mathcal{E}$-energy integral. Choose a sequence of real numbers $t_{n} \downarrow 0$ as $n \rightarrow \infty$ and let

$$
\varepsilon_{n}=\underset{x \in E}{\operatorname{esssup}} \mathbb{E}_{x}\left[A_{t_{n}}\right]
$$

Then $\varepsilon_{n} \downarrow 0$ and $\mathbb{E}_{x}\left[A_{t_{n}}\right] \leqslant \varepsilon_{n}, m$-a.e. Since, by Lemma $2.4, \mathbb{E}_{x}\left[A_{t_{n}}\right]$ is quasi-continuous,

$$
\mathbb{E}_{x}\left[A_{t_{n}}\right] \leqslant \varepsilon_{n} \quad \text { for q.e. } x \in E .
$$

Let $N_{n}$ be the exceptional set in the last line. Choose a properly exceptional set $N$ containing $\bigcup_{n} N_{n}$. Clearly

$$
\lim _{t \rightarrow 0} \sup _{x \in E \backslash N} \mathbb{E}_{x}\left[A_{t}\right]=0,
$$

which proves the corollary.

\section{Girsanov transform}

In this section, we study the Girsanov transforms of symmetric Markov processes and identify the Dirichlet forms associated with the transformed processes.

Throughout this section, we assume that $u$ is a bounded function in $\mathcal{F}_{e}$. Note that no additional condition is imposed on its energy measure $\mu_{\langle u\rangle}$ in this section. Define $\rho(x)=\mathrm{e}^{u(x)}$. In the sequel, we will use the convention for this function $\rho$ that $\widetilde{\rho}(\partial)=1$. It is easy to see that $\rho-1 \in \mathcal{F}_{e}$. Thus if we define $M^{\rho}:=M^{\rho-1}$ and $N^{\rho}:=N^{\rho-1}$, then we have Fukushima's decomposition for $\widetilde{\rho}\left(X_{t}\right)$ :

$$
\widetilde{\rho}\left(X_{t}\right)-\widetilde{\rho}\left(X_{0}\right)=M_{t}^{\rho}+N_{t}^{\rho}, \quad \mathbb{P}_{x} \text {-a.s. }
$$

Moreover,

$$
M_{t}^{\rho}=M_{t}^{\rho, c}+M_{t}^{\rho, j}+M^{\rho, k},
$$

where $M_{t}^{\rho, c}=\int_{0}^{t} \widetilde{\rho}\left(X_{s-}\right) \mathrm{d} M_{s}^{u, c}$ and

$$
\begin{aligned}
M_{t}^{\rho, j}+M_{t}^{\rho, k}= & \lim _{n \rightarrow \infty}\left(\sum_{0<s \leqslant t}\left(\widetilde{\rho}\left(X_{s}\right)-\widetilde{\rho}\left(X_{s-}\right)\right) 1_{\left\{\left|\widetilde{\rho}\left(X_{s}\right)-\widetilde{\rho}\left(X_{s-}\right)\right|>1 / n\right\}}\right. \\
& \left.-\int_{0}^{t}\left(\int_{\left\{y \in E_{\partial}:\left|\widetilde{\rho}(y)-\widetilde{\rho}\left(X_{s}\right)\right|>1 / n\right\}}\left(\widetilde{\rho}(y)-\widetilde{\rho}\left(X_{s}\right)\right) N\left(X_{s}, \mathrm{~d} y\right)\right) \mathrm{d} H_{s}\right) .
\end{aligned}
$$

Define a square integrable martingale $M$ by

$$
M_{t}=\int_{0}^{t} \frac{1}{\widetilde{\rho}\left(X_{s-}\right)} \mathrm{d} M_{s}^{\rho} .
$$

Note that $M_{t}^{c}=M_{t}^{u, c}$ and 


$$
\begin{aligned}
M_{t}-M_{t-} & =\frac{1}{\widetilde{\rho}\left(X_{t-}\right)}\left(M_{t}^{\rho}-M_{t-}^{\rho}\right) \\
& =\frac{1}{\widetilde{\rho}\left(X_{t-}\right)}\left(\widetilde{\rho}\left(X_{t}\right)-\widetilde{\rho}\left(X_{t-}\right)\right)=\frac{\widetilde{\rho}\left(X_{t}\right)}{\widetilde{\rho}\left(X_{t-}\right)}-1 .
\end{aligned}
$$

Let $L_{t}^{\rho}$ be the solution to the following SDE:

$$
L_{t}^{\rho}=1+\int_{0}^{t} L_{s-}^{\rho} \mathrm{d} M_{s} .
$$

It follows from Doleans-Dade formula (cf. [15, Theorem 9.39]) that

$$
\begin{aligned}
L_{t}^{\rho} & =\exp \left(M_{t}-\frac{1}{2}\left\langle M^{c}\right\rangle_{t}\right) \prod_{0<s \leqslant t}\left(1+M_{s}-M_{s-}\right) \mathrm{e}^{-\left(M_{s}-M_{s-}\right)} \\
& =\exp \left(M_{t}-\frac{1}{2}\left\langle M^{u, c}\right\rangle_{t}\right) \prod_{0<s \leqslant t} \frac{\widetilde{\rho}\left(X_{s}\right)}{\widetilde{\rho}\left(X_{s-}\right)} \exp \left(1-\frac{\widetilde{\rho}\left(X_{s}\right)}{\widetilde{\rho}\left(X_{s-}\right)}\right) .
\end{aligned}
$$

Note that $L_{t}^{\rho}$ is a positive local martingale and therefore a supermartingale on $[0, \infty)$. Thus

$$
\mathrm{d} \widetilde{\mathbb{P}}_{x}=L_{t} \mathrm{~d} \mathbb{P}_{x} \quad \text { on } \mathcal{M}_{t} \text { for } x \in E,
$$

defines a family of probability measures on $\left(\Omega, \mathcal{M}_{\infty}\right)$. It is known that under these new measures, $X$ is a right Markov process on $E$. We will use $\left(\widetilde{X}, \mathcal{M}, \mathcal{M}_{t}, \widetilde{\mathbb{P}}_{x}, x \in E\right)$ to denote the transformed process of $X$. Here $\widetilde{X}_{t}(\omega)=X_{t}(\omega)$ but we use $\widetilde{X}_{t}$ for emphasis when working with $\widetilde{\mathbb{P}}_{x}$.

Define

$$
\widetilde{P}_{t} f(x)=\mathbb{E}_{x}\left[L_{t}^{\rho} f\left(X_{t}\right)\right] .
$$

Before stating the next result, let us recall the the definition of time reversal operator $r_{t}$ on the path space. Given a path $\omega \in\{t<\zeta\}$, define a time-reversal operator $r_{t}$ by

$$
r_{t}(\omega)(s)= \begin{cases}\omega(t-s)_{-} & \text {for } 0 \leqslant s<t \\ \omega(0) & \text { for } s \geqslant t\end{cases}
$$

Here for $r>0, \omega(r)_{-}:=\lim _{s \uparrow r} \omega(s)$. It is known (see Lemma 4.1.2 of Theorem 9.39 [12]) that operator $r_{t}$ preserves the measure $\mathbb{P}_{m}$ on $\mathcal{M}_{t} \cap\{t<\zeta\}$.

Definition 3.1. - A continuous additive functional $A_{t}$ is called even if $A_{t} \circ r_{t}=A_{t}$ for every $t<\zeta$.

LEMMA 3.2. $-\widetilde{P}_{t}$ is symmetric on $L^{2}\left(E, \rho^{2} m\right)$.

Proof. - Let $f, g \in \mathcal{B}_{b}^{+}(E)$. By time reversal, we have

$$
\begin{aligned}
\left(\widetilde{P}_{t} f, g\right)_{\rho^{2} m} & =\left(\mathbb{E} \cdot\left[L_{t}^{\rho} f\left(X_{t}\right)\right], g\right)_{\rho^{2} m} \\
& =\mathbb{E}_{m}\left[L_{t}^{\rho} f\left(X_{t}\right) g\left(X_{0}\right) \rho^{2}\left(X_{0}\right)\right] \\
& =\mathbb{E}_{m}\left[L_{t}^{\rho} \circ r_{t} g\left(X_{t}\right) \rho^{2}\left(X_{t}\right) f\left(X_{0}\right)\right] .
\end{aligned}
$$


To show

$$
\left(\widetilde{P}_{t} f, g\right)_{\rho^{2} m}=\left(f, \widetilde{P}_{t} g\right)_{\rho^{2} m}=\mathbb{E}_{m}\left[L_{t}^{\rho} g\left(X_{t}\right) \rho^{2}\left(X_{0}\right) f\left(X_{0}\right)\right],
$$

it suffices to prove the following identity

$$
L_{t}^{\rho} \circ r_{t}=L_{t}^{\rho} \frac{\rho^{2}\left(X_{0}\right)}{\rho^{2}\left(X_{t}\right)} \quad \mathbb{P}_{m} \text {-a.s. on }\{t<\zeta\} .
$$

To this end, note that on $\{t<\zeta\}$ by (3.1),

$$
\begin{aligned}
M_{t}= & M_{t}^{u, c}+\lim _{n \rightarrow \infty}\left\{\sum_{0<s \leqslant t}\left(\frac{\widetilde{\rho}\left(X_{s}\right)}{\widetilde{\rho}\left(X_{s-}\right)}-1\right) 1_{\left\{\left|\widetilde{\rho}\left(X_{s}\right)-\widetilde{\rho}\left(X_{s-}\right)\right|>1 / n\right\}}\right. \\
& \left.-\int_{0}^{t}\left(\int_{\left\{y \in E_{\partial}:\left|\widetilde{\rho}(y)-\widetilde{\rho}\left(X_{s}\right)\right|>1 / n\right\}}\left(\frac{\widetilde{\rho}\left(X_{s}\right)}{\widetilde{\rho}\left(X_{s-}\right)}-1\right) N\left(X_{s}, \mathrm{~d} y\right)\right) \mathrm{d} H_{s}\right\},
\end{aligned}
$$

while as $u$ is bounded,

$$
\begin{aligned}
M_{t}^{u}= & M_{t}^{u, c}+\lim _{n \rightarrow \infty}\left\{\sum_{0<s \leqslant t}\left(\widetilde{u}\left(X_{s}\right)-\widetilde{u}\left(X_{s-}\right)\right) 1_{\left\{\left|\widetilde{\rho}\left(X_{s}\right)-\widetilde{\rho}\left(X_{s-}\right)\right|>1 / n\right\}}\right. \\
& \left.-\int_{0}^{t} \int_{\left\{y \in E_{\partial}:\left|\widetilde{\rho}(y)-\widetilde{\rho}\left(X_{s}\right)\right|>1 / n\right\}}\left(\widetilde{u}(y)-\widetilde{u}\left(X_{s}\right)\right) N\left(X_{s}, \mathrm{~d} y\right) \mathrm{d} H_{s}\right\}
\end{aligned}
$$

(cf. Theorem A.3.9 of [12]). It follows from (3.3) that

$$
L_{t}^{\rho}=\exp \left(M_{t}^{u}+A_{t}\right)
$$

where

$$
A_{t}=\int_{0}^{t}\left(\int_{E_{\partial}}\left(\widetilde{u}(y)-\widetilde{u}\left(X_{s}\right)+1-\mathrm{e}^{\widetilde{u}(y)-\widetilde{u}\left(X_{s}\right)}\right) N\left(X_{s}, \mathrm{~d} y\right)\right) \mathrm{d} H_{s}-\frac{1}{2}\left\langle M^{u, c}\right\rangle_{t} .
$$

Recall from [8] that continuous additive functionals of zero energy and continuous additive functionals of bounded variation are even (although it was proved in [8] for diffusions, the proof there in fact works for general symmetric Markov processes). Thus $\mathbb{P}_{m}$-a.s. on $\{t<\zeta\}$,

$$
\begin{aligned}
L_{t}^{\rho} \circ r_{t} & =\exp \left(M_{t}^{u} \circ r_{t}+A_{t} \circ r_{t}\right) \\
& =\exp \left(\widetilde{u}\left(X_{0}\right)-\widetilde{u}\left(X_{t}\right)+N_{t}^{u} \circ r_{t}+A_{t} \circ r_{t}\right) \\
& =\exp \left(M_{t}^{u}+A_{t}+2\left(\widetilde{u}\left(X_{0}\right)-\widetilde{u}\left(X_{t}\right)\right)\right) \\
& =L_{t}^{\rho} \frac{\rho^{2}\left(X_{0}\right)}{\rho^{2}\left(X_{t}\right)} .
\end{aligned}
$$

Here we used the fact that for fixed $\omega$, the discontinuous set of the sample path $X_{.}(\omega)$ is at most countable and that for fixed $t, X_{t}=X_{t-} P_{x}$-almost surely for $x \in E$. The proof is now complete. 
The following theorem was proved in Fitzsimmons [9] as Lemma 4.4 for symmetric diffusions. But its proof works for symmetric right Markov processes as well. For interested reader, an alternative proof is supplied in Appendix A of this paper, under an additional assumption that the energy measure $\mu_{\langle u\rangle}$ of $u$ is in the Kato class of $X$.

TheOREM 3.3. - Let $A_{t}$ be a PCAF of $X$ with Revuz measure $\mu$, then the Revuz measure for $A$ as a PCAF of $\widetilde{X}$ is $\rho^{2} \mu$.

THEOREM 3.4. - Let $(\widetilde{\mathcal{E}}, \widetilde{\mathcal{F}})$ be the Dirichlet form of $\widetilde{X}$ in $L^{2}\left(E, \rho^{2} m\right)$. Then $\widetilde{\mathcal{F}}=\mathcal{F}$ and for $f \in \widetilde{\mathcal{F}}$,

$$
\begin{aligned}
\widetilde{\mathcal{E}}(f, f)= & \frac{1}{2} \int_{E} \widetilde{\rho}(x)^{2} \mu_{\langle f\rangle}^{c}(\mathrm{~d} x)+\int_{E \times E \backslash d}(\widetilde{f}(x)-\widetilde{f}(y))^{2} \widetilde{\rho}(x) \widetilde{\rho}(y) J(\mathrm{~d} x \mathrm{~d} y) \\
& +\int_{E} \widetilde{f}(x)^{2} \rho(x) \kappa(\mathrm{d} x) .
\end{aligned}
$$

Proof. - Our proof uses ideas from [9] but modifications are needed since our process $X$ may have jumps and killings inside $E$. It is known (see VI.3 of [17]) that there is an $\widetilde{\mathcal{E}}$-nest $\left\{K_{n}\right\}_{n} \geqslant 1$ of compact sets and a sequence of $h_{n} \in \widetilde{\mathcal{F}}$ such that $\widetilde{h}_{n}=1$ on $K_{n}$. By the probabilistic characterization of $\mathcal{E}$-nest, $\left\{K_{n}\right\}_{n} \geqslant 1$ is an $\mathcal{E}$-nest (for process $X$ ) as well. Fix $n \geqslant 1$. For bounded $f \in \mathcal{F}_{K_{n}}=\left\{g \in \mathcal{F}: \widetilde{g}=0\right.$ q.e. on $\left.K_{n}^{c}\right\}$, the following Lyons-Zheng's forward-backward martingale decomposition holds:

$$
\tilde{f}\left(X_{t}\right)-\tilde{f}\left(X_{0}\right)=\frac{1}{2}\left(M_{t}^{f}-M_{t}^{f} \circ r_{t}\right) \quad \mathbb{P}_{m} \text {-a.s. on }\{t<\zeta\},
$$

where $M_{t}^{f}$ is the martingale part in Fukushima's decomposition (1.2) for $\widetilde{f}(X)$. Recall that $\widetilde{\mathrm{P}}_{x}=L_{t}^{\rho} \mathrm{d} \mathbb{P}_{x}$ on $\mathcal{M}_{t}$. By the Girsanov transform (see [15]),

$$
K_{t}:=M_{t}^{f}-\int_{0}^{t} \frac{1}{L_{s-}^{\rho}} \mathrm{d}\left\langle M^{f}, L^{\rho}\right\rangle_{s}=M_{t}^{f}-\left\langle M^{f}, M\right\rangle_{t}
$$

is a martingale additive functional under $\widetilde{\mathbb{P}}_{x}$ and

$$
[K]_{t}\left(\widetilde{\mathbb{P}}_{x}\right)=\left[M^{f}\right]_{t}\left(\mathbb{P}_{x}\right) \quad \widetilde{\mathbb{P}}_{x} \text {-a.s. }
$$

Here $[K]\left(\widetilde{\mathbb{P}}_{x}\right)$ is the square bracket for martingale $K$ under probability measure $\widetilde{\mathbb{P}}_{x}$ and $\left[M^{f}\right]\left(\mathbb{P}_{x}\right)$ is the square bracket for martingale $M^{f}$ under probability measure $\mathbb{P}_{x}$. We will use $\langle K\rangle\left(\widetilde{\mathbb{P}}_{x}\right)$ and $\left\langle M^{f}\right\rangle\left(\mathbb{P}_{x}\right)$ to denote the predictable dual projection of $[K]\left(\widetilde{\mathbb{P}}_{x}\right)$ and $\left[M^{f}\right]\left(\mathbb{P}_{x}\right)$ under measure $\widetilde{\mathbb{P}}_{x}$ and $\mathbb{P}_{x}$, respectively. It follows from the last identity (see, for example, [15]) that

$$
\begin{aligned}
\langle K\rangle_{t}\left(\widetilde{\mathbb{P}}_{x}\right) & =\left\langle M^{f}\right\rangle_{t}\left(\mathbb{P}_{x}\right)+\int_{0}^{t} \frac{1}{L_{s-}^{\rho}} \mathrm{d}\left\langle\left[M^{f}\right], L^{\rho}\right\rangle_{s}\left(\mathbb{P}_{x}\right) \\
& =\left\langle M^{f}\right\rangle_{t}\left(\mathbb{P}_{x}\right)+\left\langle\left[M^{f}\right], M\right\rangle_{t}\left(\mathbb{P}_{x}\right)
\end{aligned}
$$




$$
\begin{aligned}
& =\left\langle M^{f}\right\rangle_{t}\left(\mathbb{P}_{x}\right)+\left(\sum_{0<s \leqslant t}\left(\tilde{f}\left(X_{s}\right)-\tilde{f}\left(X_{s-}\right)\right)^{2}\left(\frac{\widetilde{\rho}\left(X_{s}\right)}{\widetilde{\rho}\left(X_{s-}\right)}-1\right)\right)^{p}\left(\mathbb{P}_{x}\right) \\
& =\left\langle M^{f}\right\rangle_{t}\left(\mathbb{P}_{x}\right)+\int_{0}^{t} \int_{E_{\partial}}\left(\tilde{f}\left(X_{s}\right)-\tilde{f}(y)\right)^{2}\left(\frac{\widetilde{\rho}(y)}{\widetilde{\rho}\left(X_{s}\right)}-1\right) N\left(X_{s}, \mathrm{~d} y\right) \mathrm{d} H_{s} .
\end{aligned}
$$

Thus by Theorem 3.3, the Revuz measure for the $\operatorname{PCAF}\langle K\rangle_{t}\left(\widetilde{\mathbb{P}}_{x}\right)$ of $\tilde{X}$ is

$$
\begin{aligned}
& \widetilde{\rho}(x)^{2} \mu_{\langle f\rangle}(\mathrm{d} x)+2 \widetilde{\rho}(x)^{2} \int_{E}(\widetilde{f}(x)-\widetilde{f}(y))^{2}\left(\frac{\widetilde{\rho}(y)}{\widetilde{\rho}(x)}-1\right) J(\mathrm{~d} x \mathrm{~d} y) \\
& \quad+\widetilde{f}(x)^{2} \widetilde{\rho}(x)^{2}\left(\frac{1}{\widetilde{\rho}(x)}-1\right) \kappa(\mathrm{d} x) \\
& =\widetilde{\rho}(x)^{2} \mu_{\langle f\rangle}^{c}(\mathrm{~d} x)+2 \int_{y \in E}(\widetilde{f}(x)-\widetilde{f}(y))^{2} \widetilde{\rho}(x) \widetilde{\rho}(y) J(\mathrm{~d} x \mathrm{~d} y) \\
& \quad+\widetilde{f}(x)^{2} \widetilde{\rho}(x) \kappa(\mathrm{d} x) .
\end{aligned}
$$

Note that $\left\langle M^{f}, M\right\rangle_{t}=\left\langle M^{f}, M\right\rangle_{t} \circ r_{t}$ on $\{t<\zeta\}$. So by (3.7)

$$
\widetilde{f}\left(X_{t}\right)-\tilde{f}\left(X_{0}\right)=\frac{1}{2}\left(K_{t}-K_{t} \circ r_{t}\right) \quad \mathbb{P}_{m} \text {-a.s. on }\{t<\zeta\}
$$

Let $v=\rho^{2} m$ and

$$
\widetilde{\mathbb{P}}_{v}(\cdot)=\int_{E} \widetilde{\mathbb{P}}_{x}(\cdot) v(\mathrm{~d} x) .
$$

Now applying Theorem 3.3 and noting that time reversal operator $r_{t}$ also leaves measure $\widetilde{\mathbb{P}}_{v}$ invariant on $\{t<\zeta\}$, we have by (3.9)-(3.10),

$$
\begin{aligned}
\lim _{t \rightarrow 0} & \frac{1}{t} \widetilde{\mathbb{E}}_{v}\left[\left(f\left(\widetilde{X}_{t}\right)-f\left(\widetilde{X}_{0}\right)\right)^{2} ; t<\zeta\right] \\
\leqslant & \lim _{t \rightarrow 0}\left(\frac{1}{2 t} \widetilde{\mathbb{E}}_{v}\left[\left(K_{t}\right)^{2} ; t<\zeta\right]+\frac{1}{2 t} \widetilde{\mathbb{E}}_{v}\left[\left(K_{t} \circ r_{t}\right)^{2} ; t<\zeta\right]\right) \\
= & \lim _{t \rightarrow 0} \frac{1}{t} \widetilde{\mathbb{E}}_{v}\left[\langle K\rangle_{t}(\widetilde{\mathbb{P}}) ; t<\zeta\right] \\
\leqslant & \int_{E} \widetilde{\rho}(x)^{2} \mu_{\langle f\rangle}^{c}(\mathrm{~d} x)+2 \int_{E \times E \backslash d}(\widetilde{f}(x)-\widetilde{f}(y))^{2} \widetilde{\rho}(x) \widetilde{\rho}(y) J(\mathrm{~d} x \mathrm{~d} y) \\
& +\int_{E} \widetilde{f}(x)^{2} \rho(x) \kappa(\mathrm{d} x) \\
\leqslant & 2\|\rho\|_{\infty}^{2} \mathcal{E}(f, f)<\infty .
\end{aligned}
$$

Recall that $f=0 m$-a.e. on $K_{n}^{c}$ and $h_{n} \in \widetilde{\mathcal{F}}$ with $h_{n}=1 m$-a.e. on $K_{n}$. Thus $f=f h_{n}$ and 


$$
\begin{aligned}
\lim _{t \rightarrow 0} \frac{1}{t} \int_{E}\left(f(x)-\widetilde{P}_{t} f(x)\right) f(x) v(\mathrm{~d} x)= & \lim _{t \rightarrow 0} \frac{1}{t}\left(\frac{1}{2} \widetilde{\mathbb{E}}_{v}\left[\left(f\left(\widetilde{X}_{t}\right)-f\left(\widetilde{X}_{0}\right)\right)^{2} ; t<\zeta\right]\right. \\
& \left.+\int_{E} f(x)^{2}\left(1-\widetilde{P}_{t} 1\right)(x) v(\mathrm{~d} x)\right) \\
\leqslant & \limsup _{t \rightarrow 0} \frac{1}{2 t} \widetilde{\mathbb{E}}_{v}\left[\left(f\left(\widetilde{X}_{t}\right)-f\left(\widetilde{X}_{0}\right)\right)^{2} ; t<\zeta\right] \\
& +\limsup _{t \rightarrow 0} \frac{1}{t} \int_{E}\left(f h_{n}\right)(x)^{2}\left(1-\widetilde{P}_{t} 1\right)(x) v(\mathrm{~d} x) \\
\leqslant & \limsup _{t \rightarrow 0} \frac{1}{2 t} \widetilde{\mathbb{E}}_{v}\left[\left(f\left(\widetilde{X}_{t}\right)-f\left(\widetilde{X}_{0}\right)\right)^{2} ; t<\zeta\right] \\
& +\|f\|_{\infty} \limsup _{t \rightarrow 0} \frac{1}{t} \int_{E} h_{n}(x)^{2}\left(1-\widetilde{P}_{t} 1\right)(x) v(\mathrm{~d} x) \\
\leqslant & \|\|_{\infty}^{2} \mathcal{E}(f, f)+\|f\|_{\infty} \widetilde{\mathcal{E}}\left(h_{n}, h_{n}\right)<\infty .
\end{aligned}
$$

Therefore $f \in \widetilde{\mathcal{F}}$ and so it admits a Fukushima's decomposition:

$$
\widetilde{f}\left(\widetilde{X}_{t}\right)-\widetilde{f}\left(\widetilde{X}_{0}\right)=\widetilde{M}_{t}^{f}+\widetilde{N}_{t}^{f}, \quad \widetilde{\mathbb{P}}_{\nu} \text {-a.s. }
$$

where $\widetilde{M}_{t}^{f}$ is a $\widetilde{\mathbb{P}}_{x}$-sequare integrale martingale and $\widetilde{N}_{t}^{f}$ is a continuous process of zeroenergy, particularly a continuous process of zero quadratic variation. On the other hand, $\widetilde{f}\left(X_{t}\right)$ has Fukushima's decomposition under measure $\mathbb{P}$

$$
\widetilde{f}\left(X_{t}\right)-\tilde{f}\left(X_{0}\right)=M_{t}^{f}+N_{t}^{f} .
$$

Since by Girsanov transform, $K_{t}=M_{t}^{f}-\left\langle M^{f}, M\right\rangle_{t}$ is a martingale under $\widetilde{\mathbb{P}}_{x}$, by the uniqueness of Fukushima's decomposition we have

$$
\widetilde{M}_{t}^{f}=K_{t} \quad \text { for } t \geqslant 0 .
$$

To find the expression for $\widetilde{\mathcal{E}}(f, f)$, we first calculate the killing measure $\widetilde{\kappa}$ for transformed process $\left\{\widetilde{X}, \widetilde{\mathbb{P}}_{x}, x \in E\right\} . \widetilde{\kappa}$ is the Revuz measure for $\operatorname{PCAF}\left(1_{\left\{t \geqslant \zeta_{i}\right\}}\right)^{p}(\widetilde{\mathbb{P}})$, the predictable dual projection under measure $\widetilde{\mathbb{P}}$ for nondecreasing function $t \rightarrow 1_{\left\{t \geqslant \zeta_{i}\right\}}$, where $\zeta_{i}$ is the inaccessible part of the lifetime $\zeta$ for process $X$. By the same reasoning as that in (3.8),

$$
\begin{aligned}
\left(1_{\left\{t \geqslant \zeta_{i}\right\}}\right)^{p}(\widetilde{\mathbb{P}}) & =\left(1_{\left\{t \geqslant \zeta_{i}\right\}}\right)^{p}(\mathbb{P})+\left\langle 1_{\left\{t \geqslant \zeta_{i}\right\}}, M\right\rangle_{t}(\mathbb{P}) \\
& =\left(1_{\left\{t \geqslant \zeta_{i}\right\}}\right)^{p}(\mathbb{P})+\left(\left(\frac{1}{\widetilde{\rho}\left(X_{\zeta_{i}-}\right)}-1\right) 1_{\left\{t \geqslant \zeta_{i}\right\}}\right)^{p} \\
& =\left(\widetilde{\rho}\left(X_{\zeta_{i}-}\right)^{-1} 1_{\left\{t \geqslant \zeta_{i}\right\}}\right)^{p}(\mathbb{P}) .
\end{aligned}
$$

Thus by Theorem 3.3,

$$
\widetilde{\kappa}(\mathrm{d} x)=\widetilde{\rho}(x) \kappa(\mathrm{d} x) .
$$


Now by (3.9), (3.12) and (3.13)

$$
\begin{aligned}
\widetilde{\mathcal{E}}(f, f)= & \lim _{t \rightarrow 0} \frac{1}{2 t} \widetilde{\mathbb{E}}_{v}\left[\left(f\left(\widetilde{X}_{t}\right)-f\left(\widetilde{X}_{0}\right)\right)^{2}\right]+\frac{1}{2} \int_{E} \widetilde{f}(x)^{2} \widetilde{\kappa}(\mathrm{d} x) \\
= & \lim _{t \rightarrow 0} \frac{1}{2 t} \widetilde{\mathbb{E}}_{v}\left[\left(\widetilde{M}_{t}^{f}\right)^{2}\right]+\frac{1}{2} \int_{E} \widetilde{f}(x)^{2} \widetilde{\kappa}(\mathrm{d} x) \\
= & \lim _{t \rightarrow 0} \frac{1}{2 t} \widetilde{\mathbb{E}}_{v}\left[\left\langle K_{t}\right\rangle\right]+\frac{1}{2} \int_{E} \widetilde{f}(x)^{2} \widetilde{\kappa}(\mathrm{d} x) \\
= & \frac{1}{2} \int_{E} \widetilde{\rho}(x)^{2} \mu_{\langle f\rangle}^{c}(\mathrm{~d} x)+\int_{E \times E \backslash d}(\widetilde{f}(x)-\widetilde{f}(y))^{2} \widetilde{\rho}(x) \widetilde{\rho}(y) J(\mathrm{~d} x \mathrm{~d} y) \\
& +\int_{E} \widetilde{f}(x)^{2} \rho(x) \kappa(\mathrm{d} x) .
\end{aligned}
$$

Since $f$ is an arbitrary bounded function in $\mathcal{F}_{K_{n}}$, we conclude that $\bigcup_{n \geqslant 1} \mathcal{F}_{K_{n}} \subset \widetilde{\mathcal{F}}$ and therefore $\mathcal{F} \subset \widetilde{\mathcal{F}}$. By the following theorem, we can interchange the roles of $\left(X_{t}, \mathbb{P}_{x}, x \in E\right)$ and $\left(X_{t}, \widetilde{\mathbb{P}}_{x}, x \in E\right)$ to deduce that $\widetilde{\mathcal{F}} \subset \mathcal{F}$ and hence $\widetilde{\mathcal{F}}=\mathcal{F}$.

Recall that $\rho=\mathrm{e}^{u}$ and that we take the convention that $\widetilde{\rho}(\partial)=1$. Since $u \in \mathcal{F}_{b}$, $\mathrm{e}^{-u}-1 \in \mathcal{F}_{b} \subset D(\widetilde{\mathcal{E}})$. So $\widetilde{\rho}^{-1}\left(\widetilde{X}_{t}\right)$ has Fukushima's decomposition

$$
\widetilde{\rho}^{-1}\left(\widetilde{X}_{t}\right)-\widetilde{\rho}^{-1}\left(\widetilde{X}_{0}\right)=\widetilde{M}_{t}^{\rho^{-1}}+\widetilde{N}_{t}^{\rho^{-1}}
$$

where $\widetilde{M}^{\rho^{-1}}=\widetilde{M}^{\frac{1}{\rho}-1}$ is a martingale additive functional of $\widetilde{X}$ having finite energy and $\widetilde{N}^{\rho^{-1}}=\widetilde{N}^{\frac{1}{\rho}-1}$ is a continuous additive functional of $\widetilde{X}$ having zero energy. In analogous to the definition of $M$ and $L^{\rho}$ in (3.1) and (3.2) with respect to process $X$, we define for process $\widetilde{X}$,

$$
\widetilde{M}_{t}=\int_{0}^{t} \widetilde{\rho}\left(\widetilde{X}_{s-}\right) \mathrm{d} \widetilde{M}_{s}^{\rho^{-1}}, \quad t \geqslant 0,
$$

and $\widetilde{L}^{\rho^{-1}}$ be the unique solution to

$$
\widetilde{L}^{\rho^{-1}}=1+\int_{0}^{t} \widetilde{L}_{s-}^{\rho^{-1}} \mathrm{~d} \widetilde{M}_{s}, \quad t \geqslant 0 .
$$

THEOREM 3.5. - Let $L^{\rho}$ and $\widetilde{L}^{\rho^{-1}}$ be defined by (3.2) and (3.16) respectively. Then $1 / L_{t}^{\rho}=\widetilde{L}_{t}^{1 / \rho} \mathbb{P}_{x}$-a.s. for q.e. $x \in E$.

Proof. - Since $\rho^{-1}-1 \in \mathcal{F}_{b}, \widetilde{\rho}^{-1}\left(X_{t}\right)$ has Fukushima's decomposition

$$
\widetilde{\rho}^{-1}\left(X_{t}\right)-\widetilde{\rho}^{-1}\left(X_{0}\right)=M_{t}^{\rho^{-1}}+N_{t}^{\rho^{-1}}, \quad t \geqslant 0,
$$


where $M^{\rho^{-1}}=M^{\frac{1}{\rho}-1}$ is a martingale additive functional of $X$ having finite energy and $N^{\rho^{-1}}=N^{\frac{1}{\rho}-1}$ is a continuous additive functional of $X$ having zero energy. Moreover

$$
\begin{aligned}
M_{t}^{\rho^{-1}}= & M_{t}^{\rho^{-1}, c}+M_{t}^{\rho^{-1}, j}+M^{\rho^{-1}, k} \\
= & \int_{0}^{t} \widetilde{\rho}\left(X_{s-}\right)^{-1} \mathrm{~d} M_{s}^{u, c}+\lim _{n \rightarrow \infty}\left(\sum_{0<s \leqslant t}\left(\widetilde{\rho}\left(X_{s}\right)^{-1}-\widetilde{\rho}\left(X_{s-}\right)^{-1}\right) 1_{\left\{\left|\widetilde{\rho}\left(X_{s}\right)-\widetilde{\rho}\left(X_{s-}\right)\right|>1 / n\right\}}\right. \\
& \left.-\int_{0}^{t}\left(\int_{\left\{y \in E_{\partial}:\left|\widetilde{\rho}(y)-\widetilde{\rho}\left(X_{s}\right)\right|>1 / n\right\}}\left(\widetilde{\rho}(y)^{-1}-\widetilde{\rho}\left(X_{s}\right)^{-1}\right) N\left(X_{s}, \mathrm{~d} y\right)\right) \mathrm{d} H_{s}\right)
\end{aligned}
$$

(See Theorem A.3.9 of [12] for the justification of the expression of $M_{t}^{\rho^{-1}, j}+M^{\rho^{-1}, k}$.) Thus by (3.5)

$$
\begin{aligned}
{\left[M^{\rho^{-1}}, M\right]_{t} } & =\left[M^{\rho^{-1}, c}, M^{c}\right]+\sum_{0<s \leqslant t}\left(M_{s}^{\rho^{-1}}-M_{s-}^{\rho^{-1}}\right)\left(M_{s}-M_{s-}\right) \\
& =-\int_{0}^{t} \frac{1}{\widetilde{\rho}\left(X_{s}\right)} \mathrm{d}\left\langle M^{u, c}\right\rangle_{s}+\sum_{0<s \leqslant t}\left(\frac{1}{\widetilde{\rho}\left(X_{s}\right)}-\frac{1}{\widetilde{\rho}\left(X_{s-}\right)}\right)\left(\frac{\widetilde{\rho}\left(X_{s}\right)}{\widetilde{\rho}\left(X_{s-}\right)}-1\right) \\
& =-\int_{0}^{t} \frac{1}{\widetilde{\rho}\left(X_{s}\right)} \mathrm{d}\left\langle M^{u, c}\right\rangle_{s}-\sum_{0<s \leqslant t} \frac{\left(\widetilde{\rho}\left(X_{s}\right)-\widetilde{\rho}\left(X_{s-}\right)\right)^{2}}{\widetilde{\rho}\left(X_{s}\right) \widetilde{\rho}\left(X_{s-}\right)^{2}} .
\end{aligned}
$$

Hence

$$
\begin{aligned}
\left\langle M^{\rho^{-1}}, M\right\rangle_{t}= & -\int_{0}^{t} \frac{1}{\widetilde{\rho}\left(X_{s}\right)} \mathrm{d}\left\langle M^{u, c}\right\rangle_{s} \\
& -\int_{0}^{t}\left(\int_{E_{\partial}} \frac{\left(\widetilde{\rho}(y)-\widetilde{\rho}\left(X_{s}\right)\right)^{2}}{\widetilde{\rho}(y) \widetilde{\rho}\left(X_{s}\right)^{2}} N\left(X_{s}, \mathrm{~d} y\right)\right) \mathrm{d} H_{s} .
\end{aligned}
$$

By Girsanov transform, $M^{\rho^{-1}}-\left\langle M^{\rho^{-1}}, M\right\rangle$ is a local martingale with respect to $\widetilde{\mathbb{P}}_{x}$. In view of (3.14), (3.17) and the uniqueness of Fukushima's decomposition, we have

$$
\widetilde{M}_{t}^{\rho^{-1}}=M_{t}^{\rho^{-1}}-\left\langle M^{\rho^{-1}}, M\right\rangle_{t} .
$$

So by (3.5), (3.15) and (3.18)-(3.19),

$$
\begin{aligned}
\widetilde{M}_{t} & =\int_{0}^{t} \widetilde{\rho}\left(\widetilde{X}_{s-}\right) \mathrm{d} \widetilde{M}_{s}^{\rho^{-1}} \\
& =\int_{0}^{t} \widetilde{\rho}\left(X_{s-}\right) \mathrm{d} M_{s}^{\rho^{-1}}+\left\langle M^{u, c}\right\rangle_{t}+\int_{0}^{t}\left(\int_{E_{\partial}} \frac{\left(\widetilde{\rho}(y)-\widetilde{\rho}\left(X_{s}\right)\right)^{2}}{\widetilde{\rho}(y) \widetilde{\rho}\left(X_{s}\right)} N\left(X_{s}, \mathrm{~d} y\right)\right) \mathrm{d} H_{s} \\
& =-M_{t}+\left\langle M^{u . c}\right\rangle_{t}+\sum_{0<s \leqslant t} \frac{\left(\widetilde{\rho}\left(X_{s}\right)-\widetilde{\rho}\left(X_{s-}\right)\right)^{2}}{\rho\left(X_{s}\right) \widetilde{\rho}\left(X_{s-}\right)} .
\end{aligned}
$$


This in particular imples that $\left\langle\widetilde{M}^{c}\right\rangle=\left\langle M^{u, c}\right\rangle=\left\langle M^{c}\right\rangle$. By Doleans-Dade formula and (3.3),

$$
\begin{aligned}
\widetilde{L}_{t}^{\rho^{-1}}= & \exp \left(\widetilde{M}_{t}-\frac{1}{2}\left\langle\widetilde{M}^{c}\right\rangle_{t}\right) \prod_{0<s \leqslant t} \frac{\widetilde{\rho}^{-1}\left(\widetilde{X}_{s}\right)}{\widetilde{\rho}^{-1}\left(\widetilde{X}_{s-}\right)} \exp \left(1-\frac{\widetilde{\rho}^{-1}\left(\widetilde{X}_{s}\right)}{\widetilde{\rho}^{-1}\left(\widetilde{X}_{s-}\right)}\right) \\
= & \exp \left(-M_{t}+\frac{1}{2}\left\langle M^{u, c}\right\rangle_{t}\right) \prod_{0<s \leqslant t} \exp \left(\frac{\left(\widetilde{\rho}\left(X_{s}\right)-\widetilde{\rho}\left(X_{s-}\right)\right)^{2}}{\widetilde{\rho}\left(X_{s}\right) \widetilde{\rho}\left(X_{s-}\right)}\right) \\
& \times \prod_{0<s \leqslant t} \frac{\widetilde{\rho}\left(\widetilde{X}_{s-}\right)}{\widetilde{\rho}\left(\widetilde{X}_{s}\right)} \exp \left(1-\frac{\widetilde{\rho}\left(\widetilde{X}_{s-}\right)}{\widetilde{\rho}\left(\widetilde{X}_{s}\right)}\right) \\
= & \exp \left(-M_{t}+\frac{1}{2}\left\langle M^{c}\right\rangle_{t}\right) \prod_{0<s \leqslant t} \frac{\widetilde{\rho}\left(X_{s-}\right)}{\widetilde{\rho}\left(X_{s}\right)} \exp \left(\frac{\widetilde{\rho}\left(X_{s}\right)}{\widetilde{\rho}\left(X_{s-}\right)}-1\right) \\
= & \frac{1}{L_{t}^{\rho}} .
\end{aligned}
$$

\section{Feynman-Kac type transform}

Recall that $M_{t}^{u}$ is the martingale part of the additive functional $\widetilde{u}\left(X_{t}\right)-\widetilde{u}\left(X_{0}\right)$ in the Fukushima's decomposition (1.2), $\left\langle M^{u}\right\rangle$ is the predictable dual projection of the square bracket $\left[M^{u}\right]$ of $M^{u}$, and $\mu_{\langle u\rangle}$ is the Revuz measure of PCAF $\left\langle M^{u}\right\rangle$. Throughout this section, $u$ is a function in $\mathcal{F}_{e}$ with $\mu_{\langle u\rangle}$ in Kato class of $X$. To prove Theorem 1.2, we first prepare two lemmas.

LemmA 4.1. - Suppose that $u$ is a bounded function in $\mathcal{F}_{e}$ with $\mu_{\langle u\rangle}$ in Kato class of $X$.

(i) For any real $k$,

$$
\lim _{t \downarrow 0} \underset{x \in E}{\operatorname{esssup}} \mathbb{E}_{x}\left[\exp \left(k M_{t}^{u}\right)\right]=\lim _{t \downarrow 0} \operatorname{essinf} \mathbb{E}_{x \in E}\left[\exp \left(k M_{t}^{u}\right)\right]=1 .
$$

This implies by the Markov property of $X$ that there are constants $c_{1}, c_{2}>0$ such that

$$
\underset{x \in E}{\operatorname{esssup}} \mathbb{E}_{x}\left[\exp \left(k M_{t}^{u}\right)\right] \leqslant c_{1} \exp \left(c_{2} t\right) .
$$

(ii) For any $k \geqslant 1$ and $T>0$

$$
\underset{x \in E}{\operatorname{esssup}} \mathbb{E}_{x}\left[\sup _{0 \leqslant t \leqslant T}\left(L_{t}^{\rho}\right)^{k}\right]<\infty .
$$

Hence $L_{t}^{\rho}$ is a martingale.

Proof. - (i) First note that if $|x| \leqslant M,|y| \leqslant M$, then there exists a constant $c_{M}$ such that

$$
\left|\mathrm{e}^{x}-\mathrm{e}^{y}\right| \leqslant c_{M}|x-y| \quad \text { and } \quad\left|\mathrm{e}^{x}-x-1\right| \leqslant c_{M} x^{2} .
$$


By (3.6) with $2 k u$ and $\mathrm{e}^{2 k u}$ in place of $u$ and $\rho$, we have

$$
\mathbb{E}_{x}\left[\exp \left(M^{2 k u}+A_{t}^{2 k u}\right)\right] \leqslant 1,
$$

where

$$
A_{t}^{2 k u}=\int_{0}^{t}\left(\int_{E_{\partial}}\left(2 k \widetilde{u}(y)-2 k \widetilde{u}\left(X_{s}\right)+1-\mathrm{e}^{2 k \widetilde{u}(y)-2 k \widetilde{u}\left(X_{s}\right)}\right) N\left(X_{s}, \mathrm{~d} y\right)\right) \mathrm{d} H_{s}-\frac{1}{2}\left\langle M^{2 k u, c}\right\rangle_{t} .
$$

The Revuz measure $\mu$ for continuous additive functional $A^{2 k u}$ of $X$ is

$$
\begin{aligned}
\mu(\mathrm{d} x)= & \int_{E_{\partial}}\left(2 k \widetilde{u}(y)-2 k \widetilde{u}(x)+1-\mathrm{e}^{2 \widetilde{u}(y)-2 k \widetilde{u}(x)}\right) N(x, \mathrm{~d} y) v(\mathrm{~d} x)-2 k^{2} \mu_{\langle u\rangle}^{c}(\mathrm{~d} x) \\
= & 2 \int_{E \times E \backslash d}\left(2 k \widetilde{u}(y)-2 k \widetilde{u}(x)+1-\mathrm{e}^{2 k \widetilde{u}(y)-2 k \widetilde{u}(x)}\right) J(\mathrm{~d} x, \mathrm{~d} y) \\
& +\left(1-2 k \widetilde{u}(x)-\mathrm{e}^{-2 k \widetilde{u}(x)}\right) \kappa(\mathrm{d} x)-2 k^{2} \mu_{\langle u\rangle}^{c}(\mathrm{~d} x) .
\end{aligned}
$$

Thus by (4.1) and (2.1), the total variation of measure $\mu$

$$
\begin{aligned}
|\mu|(\mathrm{d} x) & \leqslant c \int_{E \times E \backslash d}(\widetilde{u}(x)-\widetilde{u}(y))^{2} J(\mathrm{~d} x, \mathrm{~d} y)+c \widetilde{u}(x)^{2} \kappa(\mathrm{d} x)+c \mu_{\langle u\rangle}^{c}(\mathrm{~d} x) \\
& \leqslant c \mu_{\langle u\rangle}(\mathrm{d} x) .
\end{aligned}
$$

So $\mu$ and hence $A^{2 k u}$ is in the Kato class of process $X$. Now by Cauchy-Schwartz inequality and (4.2),

$$
\begin{aligned}
\mathbb{E}_{x}\left[\exp \left(k M^{u}\right)\right] & =\mathbb{E}_{x}\left[\exp \left(M_{t}^{k u}+\frac{1}{2} A_{t}^{2 k u}\right) \exp \left(-\frac{1}{2} A_{t}^{2 k u}\right)\right] \\
& \leqslant\left(\mathbb{E}_{x}\left[\exp \left(M_{t}^{2 k u}+A_{t}^{2 k u}\right)\right] \mathbb{E}_{x}\left[\exp \left(-A_{t}^{2 k u}\right)\right]\right)^{1 / 2} \\
& \leqslant\left(\mathbb{E}_{x}\left[\exp \left(-A_{t}^{2 k u}\right)\right]\right)^{1 / 2} .
\end{aligned}
$$

Hence by Corollary 2.5 and Khasminskii's lemma,

$$
\lim _{t \downarrow 0} \underset{x \in E}{\operatorname{essup}} \mathbb{E}_{x}\left[\exp \left(k M^{u}\right)\right] \leqslant 1 .
$$

On the other hand, by Jensen's inequality

$$
\mathbb{E}_{x}\left[\exp \left(k M_{t}^{u}\right)\right] \geqslant \exp \left(\mathbb{E}_{x}\left[k M_{t}^{u}\right]\right)=1 .
$$

This, together with the Markov property of $X$, proves (i).

(ii) By (3.6) $\left(L_{t}^{\rho}\right)^{k}=\exp \left(k M_{t}^{u}+k A_{t}^{u}\right)$, where $A^{u}$ is given by (4.3). Note that $2 k A^{u}$ is a continuous additive functional of $X$ whose Revuz measure is in Kato class of $X$. By Cauchy-Schwartz inequality,

$$
\mathbb{E}_{x}\left[\left(L_{t}^{\rho}\right)^{k}\right] \leqslant\left(\mathbb{E}_{x}\left[\exp \left(2 k M_{t}^{u}\right)\right] \mathbb{E}_{x}\left[\exp \left(2 k A_{t}^{u}\right)\right]\right)^{1 / 2} .
$$


It follows from part (i) of this Lemma, the Khasminskii's lemma and the Markov property of $X$ that

$$
\sup _{0 \leqslant t \leqslant T} \operatorname{esssup} \mathbb{E}_{x \in E}\left[\left(L_{t}^{\rho}\right)^{k}\right]<\infty
$$

By Doob's maximal inequailty, this implies

$$
\underset{x \in E}{\operatorname{esssup}} \mathbb{E}_{x}\left[\sup _{0 \leqslant t \leqslant T}\left(L_{t}^{\rho}\right)^{k}\right]<\infty
$$

for all $k>1$, which in particular implies that $L_{t}^{\rho}$ is a martingale.

Lemma 4.2. - Suppose that $u$ is a bounded function in $\mathcal{F}_{e}$ with $\mu_{\langle u\rangle}$ in Kato class of $X$ and that $A$ is a PCAF of $X$ whose Revuz measure measure $\mu$ is in the Kato class for $X$. Then the Revuz measure $\mathrm{e}^{2 \widetilde{u}} \mu(\mathrm{d} x)$ for A as a PCAF for the transformed process $\widetilde{X}$ is in the Kato class for $\widetilde{X}$, that is

$$
\lim _{t \downarrow 0} \underset{x \in E}{\operatorname{essup}} \widetilde{\mathbb{E}}_{x}\left[A_{t}\right]=0 .
$$

Proof. - Let $N$ be the exceptional set described in Corollary 2.5. For any $x \in E \backslash N$, by the Markov property of $X$,

$$
\begin{aligned}
\mathbb{E}_{x}\left[A_{t}^{2}\right] & =E_{x}\left[\int_{0}^{t} \int_{0}^{t} \mathrm{~d} A_{r} \mathrm{~d} A_{s}\right]=2 \mathbb{E}_{x}\left[\int_{0}^{t}\left(\int_{s}^{t} \mathrm{~d} A_{r}\right) \mathrm{d} A_{s}\right] \\
& =2 \mathbb{E}_{x}\left[\int_{0}^{t} \mathbb{E}_{X_{s}}\left[A_{t-s}\right] \mathrm{d} A_{s}\right]=2 \mathbb{E}_{x}\left[\int_{0}^{t}\left(\mathbb{E}_{X_{s}}\left[A_{t-s}\right]\right) \mathrm{d} A_{s}, X_{s} \in E \backslash N\right] \\
& \leqslant 2\left(\sup _{y \in E \backslash N} \mathbb{E}_{y}\left[A_{t}\right]\right) \mathbb{E}_{x}\left[A_{t}\right] .
\end{aligned}
$$

Hence by Corollary 2.5,

$$
\lim _{t \downarrow 0} \operatorname{esssup}_{x \in E} \mathbb{E}_{x}\left[A_{t}^{2}\right]=0 .
$$

Now

$$
\begin{aligned}
\lim _{t \downarrow 0} \underset{x \in E}{\operatorname{essup}} \widetilde{\mathbb{E}}_{x}\left[A_{t}\right] & \leqslant \lim _{t \downarrow 0} \operatorname{esssup} \mathbb{E}_{x \in E}\left[L_{t}^{\rho} A_{t}\right] \\
& \leqslant \lim _{t \downarrow 0} \operatorname{esssup}_{x \in E}\left\{\left(\mathbb{E}_{x}\left[\left(L_{t}^{\rho}\right)^{2}\right]\right)^{1 / 2}\left(\mathbb{E}_{x}\left[A_{t}^{2}\right]\right)^{1 / 2}\right\} \\
& =0 .
\end{aligned}
$$

The last equality is due to (4.5) and Lemma 4.1(ii).

Proof of Theorem 1.2 for bounded $u$. We first show that $\left\{\widehat{P}_{t}, t>0\right\}$ is a strongly continuous symmetric semigroup on $L^{2}(E, m)$.

Since $N_{t}^{u}$ is an even continuous additive functional of $X$, for $f, g \in L^{2}(E, m)$, 


$$
\begin{aligned}
\int_{E} f(x) \widehat{P}_{t} g(x) m(\mathrm{~d} x) & =\mathbb{E}_{m}\left[f\left(X_{0}\right) g\left(X_{t}\right) \mathrm{e}^{N_{t}^{u}}\right]=\mathbb{E}_{m}\left[\left(f\left(X_{0}\right) g\left(X_{t}\right) \mathrm{e}^{N_{t}^{u}}\right) \circ r_{t}\right] \\
& =\mathbb{E}_{m}\left[f\left(X_{t}\right) g\left(X_{0}\right) \mathrm{e}^{N_{t}^{u}}\right]=\int_{E} g(x) \widehat{P}_{t} f(x) m(\mathrm{~d} x) .
\end{aligned}
$$

Note that $N_{t}^{u}=\widetilde{u}\left(X_{t}\right)-\widetilde{u}\left(X_{0}\right)-M_{t}^{u}$. Thus by Lemma 4.1(i), for any $f \in L^{2}(E, m)$,

$$
\begin{aligned}
\int_{E}\left(\widehat{P}_{t} f\right)(x)^{2} m(\mathrm{~d} x) & \leqslant \int_{E} \mathbb{E}_{x}\left[\exp \left(-2 M_{t}^{u}\right)\right] \mathbb{E}_{x}\left[\left(f \mathrm{e}^{u}\right)^{2}\left(X_{t}\right)\right] \mathrm{e}^{-2 u(x)} m(\mathrm{~d} x) \\
& \leqslant c_{1} \mathrm{e}^{c_{2} t} \int_{E} f^{2}(x) m(\mathrm{~d} x) .
\end{aligned}
$$

Hence $\widehat{P}_{t}$ is a bounded operator on $L^{2}(E, m)$. For $f \in L^{2}(E, m)$, again by Lemma 4.1(i),

$$
\begin{aligned}
\lim _{t \downarrow 0} \| & \widehat{P}_{t} f-f \|_{L^{2}(E, m)} \\
\leqslant & \lim _{t \downarrow 0} \mathrm{e}^{\|u\|_{\infty}}\left\|\mathbb{E}_{x}\left[\mathrm{e}^{-M_{t}^{u}}\left(\mathrm{e}^{u} f\right)\left(X_{t}\right)\right]-\left(\mathrm{e}^{u} f\right)(x)\right\|_{L^{2}(E, m)} \\
\leqslant & \mathrm{e}^{\|u\|_{\infty}} \lim _{t \downarrow 0}\left\|\mathbb{E}_{x}\left[\left(\mathrm{e}^{u} f\right)\left(X_{t}\right)\right]-\left(\mathrm{e}^{u} f\right)(x)\right\|_{L^{2}(E, m)} \\
& +\mathrm{e}^{\|u\|_{\infty}} \lim _{t \downarrow 0}\left\|\mathbb{E}_{x}\left[\left(\mathrm{e}^{-M_{t}^{u}}-1\right)\left(\mathrm{e}^{u} f\right)\left(X_{t}\right)\right]\right\|_{L^{2}(E, m)} \\
\leqslant & \mathrm{e}^{\|u\|_{\infty}} \lim _{t \downarrow 0}\left\|P_{t}\left(\mathrm{e}^{u} f\right)-\mathrm{e}^{u} f\right\|_{L^{2}(E, m)} \\
& +\mathrm{e}^{\|u\|_{\infty}} \lim _{t \downarrow 0}\left(\int_{E} \mathbb{E}_{x}\left[\left(\exp \left(-M_{t}^{u}\right)-1\right)^{2}\right] \mathbb{E}_{x}\left[\left(\mathrm{e}^{u} f\right)^{2}\left(X_{t}\right)\right] m(\mathrm{~d} x)\right)^{1 / 2} \\
= & 0 .
\end{aligned}
$$

Therefore $\widehat{P}_{t}$ is a strongly continuous symmetric semigroup on $L^{2}(E, m)$.

Now we identify the quadratic form associated with semigroup $\widehat{P}_{t}$. For $f \in L^{2}(E, m)$, by (3.6),

$$
\begin{aligned}
\widehat{P}_{t} f(x) & =\mathbb{E}_{x}\left[f\left(X_{t}\right) \mathrm{e}^{\widetilde{u}\left(X_{t}\right)-\widetilde{u}\left(X_{0}\right)-M_{t}^{u}}\right] \\
& =\mathrm{e}^{-u(x)} \mathbb{E}_{x}\left[L_{t}^{\mathrm{e}^{-u}} \mathrm{e}^{-A_{t}^{-u}}\left(f \mathrm{e}^{u}\right)\left(X_{t}\right)\right],
\end{aligned}
$$

where $A^{-u}$ is defined by (4.3) with $2 k=-1$ there. We see from (4.4) the Revuz measure $\mu$ for continuous additive functional $A^{-u}$ of $X$ is

$$
\begin{aligned}
\mu(\mathrm{d} x)= & 2 \int_{E \times E \backslash d}\left(\widetilde{u}(x)-\widetilde{u}(y)+1-\mathrm{e}^{\widetilde{u}(x)-\widetilde{u}(y)}\right) J(\mathrm{~d} x, \mathrm{~d} y) \\
& +\left(\widetilde{u}(x)+1-\mathrm{e}^{\widetilde{u}(x)}\right) \kappa(\mathrm{d} x)-\frac{1}{2} \mu_{\langle u\rangle}^{c}(\mathrm{~d} x),
\end{aligned}
$$

which is in the Kato class of process $X$, and therefore by Lemma 4.2, the Revuz measure for $A$ as an additive functional of the transformed process $\widetilde{X}$ is in Kato class of $\widetilde{X}$. It is 
well known that $f \in \mathcal{D}(Q)$ if and only if

$$
\lim _{t \downarrow 0} \frac{1}{t} \int_{E}\left(\widehat{P}_{t} f(x)-f(x)\right) f(x) m(\mathrm{~d} x)<\infty .
$$

By (4.6), the left hand side of above equals

$$
\begin{aligned}
\lim _{t \downarrow 0} & \frac{1}{t} \int_{E}\left(\mathrm{e}^{u(x)} \widehat{P}_{t} f(x)-\left(f \mathrm{e}^{u}\right)(x)\right)\left(f \mathrm{e}^{u}\right)(x) \mathrm{e}^{-2 u(x)} m(\mathrm{~d} x) \\
\quad= & \lim _{t \downarrow 0} \frac{1}{t} \int_{E}\left(\mathbb{E}_{x}\left[L_{t}^{\mathrm{e}^{-u}} \mathrm{e}^{-A_{t}^{-u}}\left(f \mathrm{e}^{u}\right)\left(X_{t}\right)\right]-\left(f \mathrm{e}^{u}\right)(x)\right)\left(f \mathrm{e}^{u}\right)(x) \mathrm{e}^{-2 u(x)} m(\mathrm{~d} x) .
\end{aligned}
$$

Hence by Theorem 3.4 and Proposition 3.1 of [1], a bounded function $f$ in $L^{2}(E, m)$ is in $\mathcal{D}(Q)$ if and only if $f \mathrm{e}^{u} \in \mathcal{F}$, and therefore if and only if $f \in \mathcal{F}_{b}$. The latter is because both $\mathrm{e}^{u}-1$ and $\mathrm{e}^{-u}-1$ are in $\mathcal{F}$. It follows from (4.7)-(4.8), Theorems 3.4-3.5 and the Feynman-Kac formula (see [1]) that for $f \in \mathcal{F}_{b}$,

$$
\begin{aligned}
Q(f, f)= & \lim _{t \downarrow 0} \frac{1}{t} \int_{E}\left(\widehat{P}_{t} f(x)-f(x)\right) f(x) m(\mathrm{~d} x) \\
= & \widetilde{\mathcal{E}}\left(f \mathrm{e}^{u}, f \mathrm{e}^{u}\right)+\int_{E}\left(\widetilde{f} \mathrm{e}^{\widetilde{u}}(x)\right)^{2} \mathrm{e}^{-2 \widetilde{u}(x)} \mu(\mathrm{d} x) \\
= & \mathcal{E}(f, f)+\mathcal{E}\left(f^{2}, u\right) \\
& +\int_{E \times E \backslash d} \tilde{f}(x)^{2}\left(1+\widetilde{u}(x)-\widetilde{u}(y)-\mathrm{e}^{\widetilde{u}(x)-\widetilde{u}(y)}\right) J(\mathrm{~d} x, \mathrm{~d} y) \\
& -\int_{E \times E \backslash d} \widetilde{f}(y)^{2}\left(1+\widetilde{u}(y)-\widetilde{u}(x)-\mathrm{e}^{\widetilde{u}(y)-\widetilde{u}(x)}\right) J(\mathrm{~d} x, \mathrm{~d} y) \\
= & \mathcal{E}(f, f)+\mathcal{E}\left(f^{2}, u\right) .
\end{aligned}
$$

The last equality is due to estimate (4.2) and the symmetry of the jumping measure $J(\mathrm{~d} x, \mathrm{~d} y)$. This completes the proof of the theorem when $u$ is bounded.

Proof of Theorem 1.2 for general $u$. - For general $u \in \mathcal{F}_{e}$, define $u_{n}=((-n) \vee u) \wedge n$. As $u_{n} \in \mathcal{F}_{e}$, it has Fukushima's decomposition

$$
\widetilde{u}_{n}\left(X_{t}\right)=\widetilde{u}_{n}\left(X_{0}\right)+M_{t}^{u_{n}}+N_{t}^{u_{n}}, \quad t \geqslant 0 .
$$

As for each $n, u_{n}$ is a normal contraction of $u$ (that is, $\left|u_{n}\right| \leqslant|u|$ and $\left|u_{n}(x)-u_{n}(y)\right| \leqslant$ $|u(x)-u(y)|)$, we have by Theorem 3.2.2 in [12] that $\mu_{\left\langle u_{n}\right\rangle}^{c}(\mathrm{~d} x) \leqslant \mu_{\langle u\rangle}^{c}(\mathrm{~d} x)$ and so by (2.1) $\mu_{\left\langle u_{n}\right\rangle}(\mathrm{d} x) \leqslant \mu_{\langle u\rangle}(\mathrm{d} x)$. Therefore $\mu_{\left\langle u_{n}\right\rangle}$ is in Kato class of $X$ for each $n \geqslant 1$. Furthermore, from the proof of Proposition 2.2(i), one can find common constants $\alpha>1$ and $\lambda>1$ such that (2.4) holds for $Q$ and all $Q^{n}$ on $\mathcal{F}_{b}$, where the bilinear form $Q^{n}$ is defined via (2.3) with $u_{n}$ in place of $u$. If we define $\widehat{P}_{t}^{n}$ by

$$
\widehat{P}_{t}^{n} f(x)=\mathbb{E}_{x}\left[\mathrm{e}^{N_{t}^{u n}} f\left(X_{t}\right)\right], \quad f \geqslant 0,
$$


then $\left\{\widehat{P}_{t}^{n}\right\}$ is an $m$-symmetric, strongly continuous semigroup whose associated quadratic form is $\left(\mathcal{F}, Q^{n}\right)$. By (2.4), Lemma 1.3.2 and Theorem 1.3.1 in [12], we have

$$
\left\|\mathrm{e}^{-(\alpha-1) t} \widehat{P}_{t}^{n}\right\|_{2 \rightarrow 2} \leqslant 1 \quad \text { for } t>0 \text { and } n \geqslant 1 .
$$

Therefore

$$
\left\|\widehat{P}_{t}^{n}\right\|_{2 \rightarrow 2} \leqslant \mathrm{e}^{(\alpha-1) t} \quad \text { and } \quad\left\|\widehat{G}_{\alpha}^{n}\right\|_{2 \rightarrow 2} \leqslant 1,
$$

where $\widehat{G}_{\alpha}^{n}=\int_{0}^{\infty} \mathrm{e}^{-\alpha t} \widehat{P}_{t}^{n} \mathrm{~d} t$. Since $\lim _{n \rightarrow \infty} N_{t}^{u_{n}}=N^{u}$, by Fauto's lemma we have for $f \in L^{2}(E, m)$,

$$
\int_{E}\left(\widehat{P}_{t} f(x)\right)^{2} m(\mathrm{~d} x) \leqslant \liminf _{n \rightarrow \infty} \int_{E}\left(\widehat{P}_{t}^{n} f(x)\right)^{2} m(\mathrm{~d} x) \leqslant \mathrm{e}^{2(\alpha-1) t}\|f\|_{2}^{2} .
$$

Thus $\widehat{P}_{t}$, given by (1.3), is a well defined semigroup of bounded linear operators in $L^{2}(E, m)$. We show now that $\widehat{P}_{t}^{n} f \rightarrow \widehat{P}_{t} f$ weakly in $L^{2}(E, m)$. To this end, for any nonnegative $f, g \in L^{2}(E, m)$, define a finite measure $v$ on $\mathcal{M}_{t}$ by

$$
v(A):=\int_{E} \mathbb{E}_{x}\left[f\left(X_{t}\right) 1_{A}\right] g(x) m(\mathrm{~d} x) .
$$

Then

$$
\int_{E} \widehat{P}_{t}^{n} f(x) g(x) m(\mathrm{~d} x)=v\left[\mathrm{e}^{N_{t}^{u_{n}}}\right] .
$$

From (4.9) with $2 u$ in place of $u$, we have

$$
\sup _{n \geqslant 1} v\left[\mathrm{e}^{2 N_{t}^{u_{n}}}\right]=\sup _{n \geqslant 1} \int_{E} \mathbb{E}_{x}\left[\mathrm{e}^{N_{t}^{2 u_{n}}} f\left(X_{t}\right)\right] g(x) m(\mathrm{~d} x) \leqslant \mathrm{e}^{\beta_{0} t}\|f\|_{2}\|g\|_{2}<\infty
$$

for some $\beta_{0}>0$. So $\left\{\mathrm{e}^{N_{t}^{u_{n}}}, n \geqslant 1\right\}$ is uniformly integrable with respect to measure $v$ and therefore

$$
\lim _{n \rightarrow \infty} \int_{E} \widehat{P}_{t} f(x) g(x) m(\mathrm{~d} x)=\lim _{n \rightarrow \infty} v\left[\mathrm{e}^{N_{t}^{u_{n}}}\right]=v\left[\mathrm{e}^{N_{t}^{u}}\right]=\int_{E} \widehat{P}_{t} f(x) g(x) m(\mathrm{~d} x) .
$$

This proves that $\widehat{P}_{t}^{n} f$ converges weakly to $\widehat{P}_{t} f$ and consequently $\widehat{G}_{\beta}^{n} f$ converges weakly to $\widehat{G}_{\beta} f$ for any $f \in L^{2}(E, m)$ and $\beta \geqslant \alpha$. Here $\widehat{G}_{\beta} f:=\int_{0}^{\infty} \mathrm{e}^{-\beta t} \widehat{P}_{t} f \mathrm{~d} t$. We show next that $\left\{\widehat{G}_{\beta}, \beta \geqslant \alpha\right\}$ is the resolvent associated with the quadratic form $(Q, \mathcal{D}(Q))$ specified in Proposition 2.2. By (2.4) and (4.9), we have for $\beta>\alpha$,

$$
\mathcal{E}_{1}\left(\widehat{G}_{\beta}^{n} f, \widehat{G}_{\beta}^{n} f\right) \leqslant \lambda Q^{n}\left(\widehat{G}_{\beta}^{n} f, \widehat{G}_{\beta}^{n} f\right)=\lambda \int_{E} f(x) \widehat{G}_{\beta}^{n} f(x) m(\mathrm{~d} x) \leqslant c_{\beta}\|f\|_{2}^{2} .
$$


So $\left\{\widehat{G}_{\beta}^{n} f, n \geqslant 1\right\}$ is $\mathcal{E}_{1}$ bounded. After taking a subsequence if necessary, we may assume that $\widehat{G}_{\beta} f$ converges weakly to some $f_{0} \in \mathcal{F}$ and that the Cesáro mean $h_{n}:=$ $\sum_{k=1}^{n} \widehat{G}_{\beta}^{k} f / n$ converges to $f_{0}$ in Hilbert space $\left(\mathcal{F}, \mathcal{E}_{1}\right)$. Hence $f_{0}=\widehat{G}_{\beta} f$. After taking a further subsequence if necessary, we may assume, by (2.4), that (cf. Lemma 3.2.2 in [12])

$$
\lim _{n \rightarrow \infty} Q_{\beta}\left(\widehat{G}_{\beta}^{n} f, g\right)=Q_{\beta}\left(\widehat{G}_{\beta} f, g\right) \quad \text { for all } g \in \mathcal{F}
$$

Therefore

$$
\begin{aligned}
\int_{E} f(x) g(x) m(\mathrm{~d} x) & =\lim _{n \rightarrow \infty}\left[Q_{\beta}\left(\widehat{G}_{\beta}^{n} f, g\right)+Q_{\beta}^{n}\left(\widehat{G}_{\beta}^{n} f, g\right)-Q_{\beta}\left(\widehat{G}_{\beta}^{n} f, g\right)\right] \\
& =Q_{\beta}\left(\widehat{G}_{\beta} f, g\right)+\lim _{n \rightarrow \infty}\left[Q_{\beta}^{n}\left(\widehat{G}_{\beta}^{n} f, g\right)-Q_{\beta}\left(\widehat{G}_{\beta}^{n} f, g\right)\right] .
\end{aligned}
$$

Note that

$$
\begin{aligned}
\left|\int_{E} \widehat{G}_{\beta}^{n} f(x) \mu_{\left\langle u-u_{n}, g\right\rangle}^{c}(\mathrm{~d} x)\right| & =\left|\int_{\{x \in E: \widetilde{u}(x) \geqslant n\}} \widehat{G}_{\beta}^{n} f(x) \mu_{\left\langle u-u_{n}, g\right\rangle}^{c}(\mathrm{~d} x)\right| \\
& \leqslant\left(\int_{\{x \in E: \widetilde{u}(x) \geqslant n\}} \mu_{\langle g\rangle}^{c}(\mathrm{~d} x)\right)^{1 / 2}\left(\int_{E} \widehat{G}_{\beta}^{n} f(x)^{2} \mu_{\left\langle u-u_{n}\right\rangle}^{c}(\mathrm{~d} x)\right)^{1 / 2} \\
& \leqslant\left(\int_{\{x \in E: \widetilde{u}(x) \geqslant n\}} \mu_{\langle g\rangle}^{c}(\mathrm{~d} x)\right)^{1 / 2}\left(4 \int_{E} \widehat{G}_{\beta}^{n} f(x)^{2} \mu_{\langle u\rangle}^{c}(\mathrm{~d} x)\right)^{1 / 2} \\
& \leqslant c \mathcal{E}_{1}\left(\widehat{G}_{\beta}^{n} f, \widehat{G}_{\beta}^{n} f\right)^{1 / 2}\left(\int_{\{x \in E: \widetilde{u}(x) \geqslant n\}} \mu_{\langle g\rangle}^{c}(\mathrm{~d} x)\right)^{1 / 2} \\
& \rightarrow 0 \text { as } n \rightarrow \infty .
\end{aligned}
$$

In the second to the last inequality we used the fact that $u_{n}$ is a normal contraction of $u$ and hence $\mu_{\left\langle u-u_{n}\right\rangle}^{c} \leqslant 4 \mu_{\langle u\rangle}^{c}$. On the other hand,

$$
\begin{aligned}
\left|\int_{E} \tilde{g}(x) \mu_{\left\langle u-u_{n}, \widehat{G}_{\beta}^{n} f\right\rangle}^{c}(\mathrm{~d} x)\right| & \leqslant \mu_{\left\langle\widehat{G}_{\beta}^{n} f\right\rangle}^{c}(E)^{1 / 2}\left(\int_{E} \tilde{g}(x)^{2} \mu_{\left\langle u-u_{n}\right\rangle}^{c}(\mathrm{~d} x)\right)^{1 / 2} \\
& \leqslant \mathcal{E}\left(\widehat{G}_{\beta}^{n} f, \widehat{G}_{\beta}^{n} f\right)^{1 / 2}\left(4 \int_{\{x \in E: \widetilde{u}(x) \geqslant n\}} \widetilde{g}(x)^{2} \mu_{\langle u\rangle}^{c}(\mathrm{~d} x)\right)^{1 / 2} \\
& \rightarrow 0 \quad \text { as } n \rightarrow 0 .
\end{aligned}
$$

By the symmetry of the jumping measure $J(\mathrm{~d} x, \mathrm{~d} y)$,

$$
\begin{aligned}
& \left|\int_{E \times E \backslash d}\left(\left(\widetilde{g} \widehat{G}_{\beta}^{n} f\right)(x)-\left(\widetilde{g} \widehat{G}_{\beta}^{n} f\right)(y)\right)\left(\left(\widetilde{u}-\widetilde{u}_{n}\right)(x)-\left(\widetilde{u}-\widetilde{u}_{n}\right)(y)\right) J(\mathrm{~d} x, \mathrm{~d} y)\right| \\
& \quad=\mid \int_{E \times E \backslash d}\left(\left(\widetilde{u}-\widetilde{u}_{n}\right)(x)-\left(\widetilde{u}-\widetilde{u}_{n}\right)(y)\right)
\end{aligned}
$$




$$
\begin{aligned}
& \times\left(\widehat{G}_{\beta}^{n} f(x)-\widehat{G}_{\beta}^{n} f(y)\right)(\widetilde{g}(x)+\widetilde{g}(y)) J(\mathrm{~d} x, \mathrm{~d} y) \mid \\
\leqslant & \left(\int_{E \times E \backslash d}\left(\widehat{G}_{\beta}^{n} f(x)-\widehat{G}_{\beta}^{n} f(y)\right)^{2} J(\mathrm{~d} x, \mathrm{~d} y)\right)^{1 / 2} \\
& \times\left(4 \int_{E \times E \backslash d} \widetilde{g}(x)^{2}\left(\left(\widetilde{u}-\widetilde{u}_{n}\right)(x)-\left(\widetilde{u}-\widetilde{u}_{n}\right)(y)\right)^{2} J(\mathrm{~d} x, \mathrm{~d} y)\right)^{1 / 2} \\
\leqslant & 4 \mathcal{E}\left(\widehat{G}_{\beta}^{n} f, \widehat{G}_{\beta}^{n} f\right)^{1 / 2}\left(\int_{\{(x, y): \widetilde{u}(x)|\leqslant n, \widetilde{u}(y)| \leqslant n\}^{c} \backslash d} \widetilde{g}(x)^{2}(\widetilde{u}(x)-\widetilde{u}(y))^{2} J(\mathrm{~d} x, \mathrm{~d} y)\right)^{1 / 2} \\
\rightarrow & 0 \quad \text { as } n \rightarrow \infty .
\end{aligned}
$$

The last inequality is due to the fact that $u_{n}$ is a normal contraction of $u$. Also

$$
\begin{aligned}
& \left|\int_{E} \widehat{G}_{\beta}^{n} f(x) \widetilde{g}(x)\left(u-u_{n}\right)(x) \kappa(\mathrm{d} x)\right| \\
& \quad \leqslant\left(\int_{E} \widetilde{g}(x)^{2}\left(\widetilde{u}-\widetilde{u}_{n}\right)(x)^{2} \kappa(\mathrm{d} x)\right)^{1 / 2}\left(\int_{E} \widehat{G}_{\beta}^{n} f(x)^{2} \kappa(\mathrm{d} x)\right)^{1 / 2} \\
& \quad \leqslant \mathcal{E}\left(\widehat{G}_{\beta}^{n} f, \widehat{G}_{\beta}^{n} f\right)^{1 / 2}\left(\int_{\{x \in E:|\widetilde{u}(x)|>n\}} \widetilde{g}(x)^{2} \widetilde{u}(x)^{2}(\mathrm{~d} x)\right)^{1 / 2} \\
& \quad \rightarrow 0 \quad \text { as } n \rightarrow \infty .
\end{aligned}
$$

Now by (4.11)-(4.14),

$$
\begin{aligned}
Q_{\beta}^{n}( & \left.\widehat{G}_{\beta}^{n} f, g\right)-Q_{\beta}\left(\widehat{G}_{\beta}^{n} f, g\right) \\
= & \frac{1}{2} \int \widehat{G}_{\beta}^{n} f(x) \mu_{\left\langle u-u_{n}, g\right\rangle}^{c}(\mathrm{~d} x)+\frac{1}{2} \int \widetilde{g}(x) \mu_{\left\langle u-u_{n}, \widehat{G}_{\beta}^{n} f\right\rangle}^{c}(\mathrm{~d} x) \\
& +\int_{E \times E \backslash d}\left(\left(\widetilde{g} \widehat{G}_{\beta}^{n} f\right)(x)-\left(\widetilde{g} \widehat{G}_{\beta}^{n} f\right)(y)\right)\left(\left(\widetilde{u}-\widetilde{u}_{n}\right)(x)-\left(\widetilde{u}-\widetilde{u}_{n}\right)(y)\right) J(\mathrm{~d} x, \mathrm{~d} y) \\
& +\int \widehat{G}_{\beta}^{n} f(x) \widetilde{g}(x)\left(u-u_{n}\right)(x) \kappa(\mathrm{d} x) \\
& \rightarrow 0 \quad \text { as } n \rightarrow \infty .
\end{aligned}
$$

This, together with (4.10) shows that

$$
Q_{\beta}\left(\widehat{G}_{\beta}, g\right)=\int_{E} f(x) g(x) m(\mathrm{~d} x) \quad \text { for all } f, g \in L^{2}(E, m) .
$$

So $\widehat{G}_{\beta}$ is the resolvent associated with $(Q, \mathcal{D}(Q))$ and hence $\widehat{P}_{t}$ is its associated semigroup. Theorem 1.2 is now proved in its full generality. 
COROLLARY 4.3. - In addition to the conditions in Theorem 1.2, assume further that $u$ is bounded, continuous and that the semigroup $\left\{P_{t}\right\}_{t \geqslant 0}$ for process $X$ is strongly Feller, that is, $P_{t}$ maps $B_{b}(E)$ into $C_{b}(E)$. Then the semigroup $\left\{\widehat{P}_{t}\right\}_{t \geqslant 0}$ is also strongly Feller.

Proof. - Let $f \in B_{b}(E)$. Set $g(x)=f(x) \mathrm{e}^{u(x)}$. We have

$$
\mathrm{e}^{u(x)} \widehat{P}_{t} f(x)=\mathbb{E}_{x}\left[\exp \left(M_{t}^{-u}\right) g\left(X_{t}\right)\right] .
$$

Thus we only need to show that $\mathbb{E}_{x}\left[\exp \left(M_{t}^{-u}\right) g\left(X_{t}\right)\right]$ is continuous. Note that for any $\varepsilon \leqslant t$,

$$
\mathbb{E}_{x}\left[\exp \left(M_{t}^{-u} \circ \theta_{\varepsilon}\right) g\left(X_{t}\right)\right]=\mathbb{E}_{x}\left[\mathbb{E}_{X_{\varepsilon}}\left[g\left(X_{t-\varepsilon}\right) \exp \left(M_{t}^{-u}\right)\right]\right]
$$

is continuous by the strong Feller property of $P_{t}$. It is sufficient to prove that $\mathbb{E}_{x}\left[\exp \left(M_{t}^{-u} \circ \theta_{\varepsilon}\right) g\left(X_{t}\right)\right]$ converges to $\mathbb{E}_{x}\left[\exp \left(M_{t}^{-u}\right) g\left(X_{t}\right)\right]$ uniformly with respect to $x$ as $\varepsilon \rightarrow 0$. This is a consequence of

$$
\limsup _{\varepsilon \rightarrow 0} \mathbb{E}_{x \in E}\left[\left(\exp \left(M_{t+\varepsilon}^{-u}-M_{t}^{-u}-M_{\varepsilon}^{-u}\right)-1\right)^{4}\right]=0,
$$

which can be shown in a similar way as that of Lemma 4.1(i).

\section{Appendix}

In this appendix, we supply an alternative proof to Theorem 3.3 for bounded $u \in \mathcal{F}_{e}$ with $\mu_{\langle u\rangle}$ in Kato class of $X$.

LEMMA A.1. - Let $\mu_{n}, n \geqslant 1$ be a sequence of smooth measures of finite $\mathcal{E}$-energy integral with $\sup _{n} \mu_{n}(E)<\infty$. Assume that there is a compact subset $K \subset E$ such that $\mu_{n}\left(K^{c}\right)=0$ for $n \geqslant 1$ and that $U_{1} \mu_{n}$ converges weakly to $U_{1} \mu$ in Hilbert space $\left(\mathcal{F}, \mathcal{E}_{1}\right)$. Then for any bounded quasi-continuous function $f$,

$$
\lim _{n \rightarrow \infty} \int_{E} f(x) \mu_{n}(\mathrm{~d} x)=\int_{E} f(x) \mu(\mathrm{d} x) .
$$

Proof. - By the assumption and the regular property of the Dirichlet form, we see that $\mu_{n}$ converges to $\mu$ vaguely, i.e.,

$$
\lim _{n \rightarrow \infty} \int_{E} g(x) \mu_{n}(\mathrm{~d} x)=\int_{E} g(x) \mu(\mathrm{d} x)
$$

for any $g \in C_{c}(E)$.

Put $M=\|f\|_{\infty}$. Since $f$ is quasi-continuous, there exists an $\mathcal{E}$-nest $\left\{K_{m}, m \geqslant 1\right\}$ consisting of compact sets such that $\left.f\right|_{K_{m}}$ is continuous. By Tietz extension theorem, for each $m$, we can find a $\widehat{f}_{m}$ in $C_{c}(E)$ such that $\left.f\right|_{K_{m}}=\left.\widehat{f}\right|_{K_{m}}$ and $\left\|\widehat{f}_{m}\right\|_{\infty} \leqslant M$. Denote by $e_{m}$ the 1-potential of $K \backslash K_{m}$. Then $\operatorname{Cap}_{1}\left(K \backslash K_{m}\right)=\mathcal{E}_{1}\left(e_{m}, e_{m}\right) \rightarrow 0$ as $m \rightarrow \infty$. Now for any $m \geqslant 1$, 


$$
\begin{aligned}
& \left|\int_{E} f(x) \mu_{n}(\mathrm{~d} x)-\int_{E} f(x) \mu(\mathrm{d} x)\right| \\
& \leqslant\left|\int_{E}\left(f(x)-\widehat{f}_{m}\right) \mu_{n}(\mathrm{~d} x)\right|+\left|\int_{E} \widehat{f}_{m}(x) \mu_{n}(\mathrm{~d} x)-\int_{E} \widehat{f}_{m}(x) \mu(\mathrm{d} x)\right| \\
& \quad+\left|\int_{E}\left(f(x)-\widehat{f}_{m}(x)\right) \mu(\mathrm{d} x)\right| \\
& \leqslant 2 M \int_{K \backslash K_{m}} \mu_{n}(\mathrm{~d} x)+\left|\int_{E} \widehat{f}_{m}(x) \mu_{n}(\mathrm{~d} x)-\int_{E} \widehat{f}_{m}(x) \mu(\mathrm{d} x)\right|+2 M \int_{E \backslash K_{m}} \mu(\mathrm{d} x) \\
& \leqslant 2 M \int_{E} e_{m}(x) \mu_{n}(\mathrm{~d} x)+2 M \int_{E} e_{m}(x) \mu(\mathrm{d} x)+\left|\int_{E} \widehat{f_{m}}(x) \mu_{n}(\mathrm{~d} x)-\int_{E} \widehat{f_{m}}(x) \mu(\mathrm{d} x)\right| \\
& \leqslant 4 M \sqrt{\sup _{n} \mathcal{E}_{1}\left(U_{1} \mu_{n}, U_{1} \mu_{n}\right)} \sqrt{\operatorname{Cap}_{1}\left(K \backslash K_{m}\right)} \\
& \quad+\left|\int_{E} \widehat{f_{m}}(x) \mu_{n}(\mathrm{~d} x)-\int_{E} \widehat{f_{m}}(x) \mu(\mathrm{d} x)\right| .
\end{aligned}
$$

The lemma follows by first passing $n \rightarrow \infty$ and then $m \rightarrow \infty$.

We now give an alternative proof for Theorem 3.3.

THEOREM A.2. - Let $A_{t}$ be a PCAF of $X$ with Revuz measure $\mu$, then for any bounded function $\psi \in L^{1}(E, m)$,

$$
\widetilde{\mathbb{E}}_{\psi \rho^{2} m}\left[A_{t}\right]=\int_{E}\left(\int_{0}^{t} \widetilde{P}_{s} \psi \mathrm{d} s\right) \widetilde{\rho}^{2} \mu(\mathrm{d} x) .
$$

Therefore the Revuz measure for A as a PCAF of $\tilde{X}$ is $\rho^{2} \mu$.

Proof. - We follow the path in the proof of Lemma 6.3.6 in [12] but with some improvements. First we note that if $\phi_{k}$ converges to $\phi$ in $\left(\mathcal{F}, \mathcal{E}_{1}\right)$, then by Fukushima's decomposition,

$$
\begin{aligned}
& \left(\mathbb{E}_{m}\left(N_{t}^{\phi_{k}}-N_{t}^{\phi}\right)^{2}\right)^{1 / 2} \\
& \quad \leqslant\left\|\phi_{k}-\phi\right\|_{2}+\left(\mathbb{E}_{m}\left(\widetilde{\phi}_{k}\left(X_{t}\right)-\widetilde{\phi}\left(X_{t}\right)\right)^{2}\right)^{1 / 2}+\left(\mathbb{E}_{m}\left(M_{t}^{\phi_{k}}-M_{t}^{\phi}\right)^{2}\right)^{1 / 2} \\
& \quad \leqslant 2\left\|\phi_{k}-\phi\right\|_{2}+2 t \sqrt{\mathcal{E}\left(\phi_{k}-\phi, \phi_{k}-\phi\right)} \\
& \quad \rightarrow 0 \text { as } k \rightarrow \infty .
\end{aligned}
$$

Since $\mu$ is a smooth measure, there is an $\mathcal{E}$-nest $\left\{F_{n}\right\}_{n} \geqslant 1$ of compact sets such that $\mu\left(F_{n}\right)<\infty, 1_{F_{n}} \mu$ is of finite $\mathcal{E}$-energy integral, and $U_{1}\left(1_{F_{n}} \mu\right)$ is bounded for each $n \geqslant 1$ (see Theorem 2.2.4 of [12]). Let $g_{k}^{(n)}=k\left(U_{1}\left(1_{F_{n}} \mu\right)-k G_{k+1}\left(U_{1}\left(1_{F_{n}} \mu\right)\right)\right)$. Let $f_{n} \in \mathcal{F} \cap C_{c}(E)$ be such that $0 \leqslant f_{n} \leqslant 1$ and $f_{n}=1$ on $F_{n}$. Then for any $v \in \mathcal{F}$, 


$$
\begin{aligned}
\lim _{k \rightarrow \infty} \mathcal{E}_{1}\left(G_{1}\left(f_{n} g_{k}^{(n)}\right), v\right) & =\lim _{k \rightarrow \infty} \int_{E} g_{k}^{(n)} f_{n} v m(\mathrm{~d} x)=\int_{E} \widetilde{v} \widetilde{f}_{n} 1_{F_{n}} \mu(\mathrm{d} x) \\
& =\int_{E} \widetilde{v} 1_{F_{n}} \mu(\mathrm{d} x)=\mathcal{E}_{1}\left(U_{1}\left(1_{F_{n}} \mu\right), v\right) .
\end{aligned}
$$

So $G_{1}\left(f_{n} g_{k}^{(n)}\right)$ converges $\mathcal{E}$-weakly to $U_{1}\left(1_{F_{n}} \mu\right)$ as $k \rightarrow \infty$. By taking a Cesàro mean of a subsequence of $g_{k}^{(n)}$, there is a sequence $h_{k}^{(n)}$ in $\mathcal{F}$ such that $G_{1}\left(f_{n} h_{k}^{(n)}\right)$ converges to $U_{1}\left(1_{F_{n}} \mu\right)$ in $\left(\mathcal{F}, \mathcal{E}_{1}\right)$ as $k \rightarrow \infty$. Hence by (A.1),

$$
\lim _{k \rightarrow \infty} \mathbb{E}_{m}\left(N_{t}^{G_{1}\left(f_{n} h_{k}^{(n)}\right)}-N_{t}^{U_{1}\left(1_{F_{n}} \mu\right)}\right)^{2}=0
$$

Note that

$$
N_{t}^{G_{1}\left(f_{n} h_{k}^{(n)}\right)}=\int_{0}^{t} G_{1}\left(f_{n} h_{k}^{(n)}\right)\left(X_{s}\right) \mathrm{d} s-\int_{0}^{t}\left(f_{n} h_{k}^{(n)}\right)\left(X_{s}\right) \mathrm{d} s
$$

and

$$
N_{t}^{U_{1}\left(1_{F_{n}} \mu\right)}=\int_{0}^{t} \widetilde{U}_{1}\left(1_{F_{n}} \mu\right)\left(X_{s}\right) \mathrm{d} s-\int_{0}^{t} 1_{F_{n}}\left(X_{S}\right) \mathrm{d} A_{s}
$$

(cf. Lemma 5.4.1 of [12]). By (A.1) and the triangular inequality, we see that

$$
\lim _{k \rightarrow \infty} \mathbb{E}_{m}\left(\int_{0}^{t}\left(f_{n} h_{k}^{(n)}\right)\left(X_{s}\right) \mathrm{d} s-\int_{0}^{t} 1_{F_{n}}\left(X_{s}\right) \mathrm{d} A_{s}\right)^{2}=0
$$

Now for any bounded function $\psi \in L^{1}(E, m)=L^{1}\left(E, \rho^{2} m\right)$, by Cauchy-Schwartz inequality and (4.1),

$$
\begin{aligned}
& \lim _{k \rightarrow \infty} \widetilde{\mathbb{E}}_{\psi \rho^{2} m}\left|\int_{0}^{t}\left(f_{n} h_{k}^{(n)}\right)\left(X_{s}\right) \mathrm{d} s-\int_{0}^{t} 1_{F_{n}}\left(X_{s}\right) \mathrm{d} A_{s}\right| \\
& \quad \leqslant \lim _{k \rightarrow \infty}\left(\mathbb{E}_{\psi \rho^{2} m}\left[\left(L_{t}^{\rho}\right)^{2}\right]\right)^{1 / 2}\left(\mathbb{E}_{\psi \rho^{2} m}\left(\int_{0}^{t}\left(f_{n} h_{k}^{(n)}\right)\left(X_{s}\right) \mathrm{d} s-\int_{0}^{t} 1_{F_{n}}\left(X_{s}\right) \mathrm{d} A_{s}\right)^{2}\right)^{1 / 2} \\
& \quad=0
\end{aligned}
$$

Note that since

$$
\int_{E} g_{k}^{(n)}(x) m(\mathrm{~d} x)=\int_{E} k\left(G_{1}-k G_{1} G_{k+1 Z} 1\right) 1_{F_{n}} \mu(\mathrm{d} x)=\int_{F_{n}} k G_{k+1} 1 m u(\mathrm{~d} x) \leqslant \mu\left(F_{n}\right),
$$

$\sup _{k \geqslant 1} \int f_{n} h_{k}^{(n)} m(\mathrm{~d} x)<\infty$. Thus 


$$
\begin{aligned}
\widetilde{\mathbb{E}}_{\psi \rho^{2} m}\left[A_{t}\right] & =\lim _{n \rightarrow \infty} \widetilde{\mathbb{E}}_{\psi \rho^{2} m}\left[\int_{0}^{t} 1_{F_{n}}\left(\widetilde{X}_{s}\right) \mathrm{d} A_{s}\right] \\
& =\lim _{n \rightarrow \infty} \lim _{k \rightarrow \infty} \widetilde{\mathbb{E}}_{\psi \rho^{2} m}\left[\int_{0}^{t}\left(f_{n} h_{k}^{(n)}\right)\left(\widetilde{X}_{s}\right) \mathrm{d} s\right] \\
& =\lim _{n \rightarrow \infty} \lim _{k \rightarrow \infty} \int_{E}\left(\int_{0}^{t} \widetilde{P}_{s} \psi \mathrm{d} s\right) \rho^{2} f_{n} h_{k}^{(n)} m(\mathrm{~d} x) . \\
& =\lim _{n \rightarrow \infty} \int_{E}\left(\int_{0}^{t} \widetilde{P}_{s} \psi \mathrm{d} s\right) \rho^{2} 1_{F_{n}} \mu(\mathrm{d} x)=\iint_{E}\left(\int_{0}^{t} \widetilde{P}_{s} \psi \mathrm{d} s\right) \widetilde{\rho}^{2} \mu(\mathrm{d} x) .
\end{aligned}
$$

In the second to the last equality, we used Lemma A.2 and the fact that $\widetilde{P}_{s} \psi$ is quasicontinuous with respect to $X$ as well. The latter is due to the fact that $L_{t}^{\rho}$ is strictly positive up to lifetime $\zeta$ of $X$ and that $\widetilde{P}_{s} \psi$ is quasi-continuous with respect to $\widetilde{X}$.

\section{Acknowledgement}

We thank Len Gross for helpful discussions on Tietz extension theorem. Thanks are also due to R. Song for pointing out an error in a previous version of this paper.

\section{REFERENCES}

[1] Albeverio S., Ma Z.-M., Perturbation of Dirichlet forms-lower semiboundedness, closability, and form cores, J. Funct. Anal. 99 (1991) 332-356.

[2] Albeverio S., Röckner M., Zhang T., Girsanov transform for symmetric diffusion with infinite dimensional state space, Ann. Probab. 21 (1993) 961-978.

[3] Blumenthal R.M., Getoor R.K., Markov Processes and Potential Theory, Academic Press, New York, 1968.

[4] Chen Z.-Q., Ma Z.-M., Röckner M., Quasi-homeomorphisms of Dirichlet forms, Nagoya Math. J. 136 (1994) 1-15.

[5] Chung K.L., Zhao Z., From Brownian Motion to Schrödinger's Equation, Springer, New York, 1995.

[6] Dellacherie C., Meyer P.-A., Probabilités et Potentiel, Chapites V à VIII, Hermann, 1980.

[7] Either S.N., Kurtz T.G., Markov Processes-Characterization and Convergence, Wiley, New York, 1986.

[8] Fitzsimmons P.J., Even and odd continuous additive functionals, in: Dirichlet Forms and Stochastic Processes, De Gruyter, Berlin, 1988, pp. 139-154.

[9] Fitzsimmons P.J., Absolute continuity of symmetric diffusions, Ann. Probab. 25 (1997) $230-258$.

[10] Fitzsimmons P.J., Getoor R.K., Limit theorems and variation properties for fractional derivatives of the local time of a stable processes, Ann. Inst. Henri. Poincarè 28 (1992) 311-333.

[11] Fukushima M., On absolute continuity of multi-dimensional symmetrizalle diffusion, in: Functional Analysis in Markov Processes, Lect. Notes Math., Vol. 923, 1982, pp. 146176. 
[12] Fukushima M., Oshima Y., Takeda M., Dirichlet Forms and Symmetric Markov Processes, Walter de Gruyter, Berlin, 1994.

[13] Fukushima M., Takeda M., A transformation of symmetric Markov processes and the Donsker-Varadhan theory, Osaka J. Math. 21 (1984) 311-326.

[14] Glover J., Rao M., Sikic H., Song R., Quadratic forms corresponding to the generalized Schrödinger semigroups, J. Funct. Anal. 125 (1994) 358-378.

[15] He S.W., Wang J.G., Yan J.A., Semimartingale Theory and Stochastic Calculus, Science Press, Beijing, 1992.

[16] Kunita H., Absolute continuity of Markov processes, in: Seminaire de Probabilites X, Lect. Notes Math., Vol. 511, 1976, pp. 44-77.

[17] Ma Z.-M., Röckner M., Introduction to the Theory of (Non-symmetric) Dirichlet Forms, Springer, Berlin, 1992.

[18] Orey S., Conditions for the absolute continuity of two diffusions, Trans. Amer. Math. Soc. 193 (1974) 413-426.

[19] Oshima Y., On absolute continuity of two symmetric diffusion processes, in: Lect. Notes Math., Vol. 1250, Springer, Berlin, 1987, pp. 184-194.

[20] Oshima Y., Takeda M., On a transformation of symmetric Markov processes and recurrence property, in: Lect. Notes Math., Vol. 1250, Springer, Berlin, 1987, pp. 171-183.

[21] Sharpe M., General Theory of Markov Processes, Academic Press, 1988.

[22] Simon B., Schrödinger semigroups, Bull. Amer. Math. Soc. 7 (1982) 447-526.

[23] Takeda M., Topics on Dirichlet forms and symmetric Markov processes, Sugaku Expositions 12 (1999) 201-222.

[24] Takeda M., Zhang T., Asymptotic properties of additive functionals of Brownian motion, Ann. Probab. 25 (1997) 940-952.

[25] Zhang T., Generalized Feynman-Kac semigroups, associated quadratic forms and asymptotic properties, Preprint, 1998. To appear in Potential Analysis.

[26] Yamada T., On the fractional derivative of Brownian local time, J. Math. Kyoto Univ. 25 (1985) 49-58.

[27] Yamada T., On some limit theorems for occupation times of one dimensional Brownian motion and its continuous additive functionals locally of zero energy, J. Math. Kyoto Univ. 26 (1986) 309-322.

[28] Zhao Z., A probabilistic principle and generalized Schrödinger perturbation, J. Funct. Anal. 101 (1991) 162-176. 